\title{
Individual Differences in the Effects of Physical Activity on Classroom Behaviour
}

\section{Christina Hubertina Helena Maria Heemskerk*, Mirna Sumatic, Steve Strand and Lars-Erik Malmberg}

Department of Education, University of Oxford, Oxford, United Kingdom

Background: Promoting physical activity (PA) in children is associated with a wide range of desired outcomes. With children increasingly not meeting recommended levels of activity, the school setting offers many opportunities to improve this. Increasing children's on-task behaviour is of particular importance to teachers, and while it has previously been suggested that PA can improve classroom behaviour, a consensus on the dose-response relationship of PA content, and its interaction with children's individual differences, is yet to be reached. This study aimed to investigate this relationship more closely and assess the

OPEN ACCESS

Edited by:

Esther Hartman,

University Medical Center Groningen,

Netherlands

Reviewed by:

Spyridoula Vazou,

lowa State University, United States Juan De Dios Benítez Sillero,

University of Cordoba, Spain

*Correspondence: Christina Hubertina Helena Maria Heemskerk

christina.heemskerk@unibe.ch

Specialty section:

This article was submitted to Educational Psychology, a section of the journal

Frontiers in Education

Received: 10 November 2021 Accepted: 24 December 2021 Published: 09 February 2022

Citation:

Heemskerk CHHM, Sumatic M,

Strand $S$ and

Malmberg L-E (2022) Individual Differences in the Effects of Physical Activity on Classroom Behaviour. Front. Educ. 6:812801. doi: 10.3389/feduc.2021.812801 differences between objective and subjective measures of PA intensity.

Method: Data was collected from 76 primary school-aged children $(M$ age $=9.3$ years, $S D$ $=0.7$ years; 46 females). The PE lesson intervention followed a 3 (intensity: low, medium, high) by 2 (complexity: low, high) within-person design. Children's task-behaviour was observed pre- and post-the intervention PE lesson during "business as usual" classroom lesson. PA was measured objectively with wrist-worn accelerometer devices for $24 \mathrm{~h}$ before the intervention, and subjectively rated on a five-point Likert scale after each intervention lesson.

Results: The results indicated a difference in subjective and objective measures of PA intensity on children's on-task behaviour. Objective measures positively predicted taskbehaviour at moderate to high intensities, whereas subjective ratings were beneficial only at sub-maximal intensity. Findings suggested that boys' on-task behaviour improved at higher intensities, whereas girls were also responsive to lower intensity lessons. Less active children showed more improvement in on-task behaviour after a range of lessons, whereas more active children only benefited from the highest intensity lessons. Finally, children exhibiting the highest levels of off-task behaviour improved their on-task behaviour after all intervention lessons.

Conclusion: The findings suggest that higher intensities of PA have a generally positive effect on children's subsequent behaviour, although certain individual characteristics make children more responsive to lower PA intensities. Furthermore, passive off-task behaviours were less prevalent after lower-intensity PA. Thus, individual differences, as well as the target behaviour, are important factors to take into account when designing optimal PE lessons for improving classroom behaviour.

Keywords: physical activity, physical education, objective intensity, subjective intensity, classroom behaviour 


\section{INTRODUCTION}

Children and adolescents around the world are not meeting the minimum daily requirement of moderate-to-vigorous physical activity (MVPA), with only one in four children aged 11 being sufficiently active in 2017/18 (Inchley et al., 2020), and a declining trend from early adolescence into early adulthood (van Sluijs et al., 2021). Physical activity (PA) is widely accepted to be beneficial to physical, mental, and cognitive health, both during childhood and into later life (van Sluijs et al., 2021), making PA behaviour in youth a crucial target for interventions. The school environment is widely accepted to be a fitting setting to implement PA. Especially as many children are limited in their opportunities for exercise available to them, school becomes the ideal environment to ensure that children have the opportunity to partake in PA (Mahar, 2011). Yet, recent meta-analyses show that $\mathrm{PA}$ interventions in schools fail to reach the desired outcome of increasing activity levels in youth, and especially in girls (Owen et al., 2017; van Sluijs et al., 2021). Coupled with a trend in schools for decreasing the time allocated to breaks and PE lessons, increasing PA participation in schools is an imperative topic to investigate. It is vital to develop an intervention that will successfully engage students in the activity while also yielding the desired outcomes across physical, mental, and cognitive health domains. We investigated the dose-response relationship between the PA content of PE lessons (complexity, objective, and subjective intensity-the dose) and subsequent task-behaviour in primary school classrooms (the response), and whether this relationship differed based on the individual characteristics of the participants (BMI, sex, habitual PA, and baseline task-related behaviour). We posed the following research questions:

1) Is there a difference between the effects of subjective and objective measures of PA intensity on on-task behaviour?

2) Does the response to PA differ for passive and active off-task behaviours?

3) Are there specific sub-samples of participants who respond more or less favourably to PA? And if so, which subgroups and which PA parameters?

We hypothesised that 1) higher intensity PA would have the greatest positive effect (Ma et al., 2014), and that this response would not differ between subjective and objective measures of PA. Based on the cognitive-energetic model (Unsworth \& Robinson, 2020), we expected that 2) active off-task behaviours would be most strongly reduced after medium and high intensity PA. Yet, for passive off-task behaviour, we expected that medium, but not high intensity PA would lead to the greatest reduction, as high intensity PA may induce tiredness rather than a state of activation. Finally, we expected 3) more positive effects for all PA types and intensities for children who have lower baseline ontask behaviour (Mahar et al., 2006), for boys (van Sluijs et al., 2021), and for those who are more regularly active (Jäger et al., 2015). We did not expect to find effects of BMI (Martin et al., 2018).
To increase PA levels in children, Beets et al. (2016) suggest the theory of expanded, extended, and enhanced opportunities for physical activity. Although this theory is not limited to PA opportunities in schools, all three strategies are applicable to the school setting. Expanding involves increasing the frequency of MVPA opportunities (i.e., more breaks or PE lessons), extending means increasing the duration of MVPA opportunities (i.e., longer breaks), and enhancing is improving the quality of the PA opportunities available to increase the amount of MVPA (i.e., reducing time spent listening to instructions, quicker transitions between tasks). The PA opportunities in schools need to be of high quality, implementing developmentally appropriate activities which children will benefit from the most: improving their physical, mental, and cognitive health, including behaviour, executive functions and other developmental outcomes. Moreover, these interventions need to be designed for sustained, large-scale implementation (van Sluijs et al., 2021).

\subsection{PA and Learning Behaviour}

There is increasing evidence that behavioural engagement in the classroom, operationalised as time-on-task or on-task behaviour, is improved by PA (Masini et al., 2020). Studies in both preadolescents (e.g., De Greeff et al., 2016) and adolescents (e.g. Kubesch et al., 2009; Mavilidi et al., 2021) indicate that attention and on-task behaviour are positively affected by PA. Although results are heterogeneous, for example Goh et al. (2018) finding no significant improvement in on-task behaviour postintervention, the majority of studies investigating on-task behaviour in primary school classrooms have found positive effects of PA on time-on-task (e.g., De Greeff et al., 2018; Masini et al., 2020). This improvement in task-focus has been attributed to the break from school work provided by a physical activity (Mavilidi et al., 2019), improved executive functions, including inhibition (Vogan et al., 2018; Mahar, 2019), and improvements in affective state (Lubans et al., 2016; Burns et al., 2017). The focus in the wider literature appears to be on improving on-task behaviour, while reduction in off-task behaviour is implied, and less often measured or reported explicitly. Literature on the specific nature of off-task behaviours (passive or active) in relation to PA is scarce and this study contributes to this field by not only investigating the effects of PA on on-task behaviour, but also on passive and active off-task behaviours separately. Ma et al. (2014) found that both active and passive off-task behaviour in 2nd and 4th grade pupils was reduced after PA breaks. Active off-task behaviours, in particular motor behaviour, were reduced more greatly than passive off-task behaviours $(\mathrm{ES}=0.31-0.45$ vs $\mathrm{ES}=0.48-1.08)$ in their study (Ma et al., 2014). On the contrary, Snyder et al. (2017) found a significant reduction in passive off-task behaviour in their intervention classroom compared with the control classroom, and no significant effect for off-task active behaviours in the intervention group. Therefore, some ambiguity in the findings is present and this emphasises the need to further investigate and differentiate off-task behaviours. A common characteristic of numerous studies is the nature of the interventions being classroom-based (e.g., Goh et al., 2016; 
Snyder et al., 2017; Goh et al., 2018; Maykel et al., 2018), whereas the present study aims to assess a purposefully designed PE lesson intervention instead. Therefore, this is a further gap in the literature that we aim to contribute to.

\subsubsection{Physical Activity Intensity}

Vigorous PA is important to physical health. For example, Buchan et al. (2011) found moderate and high intensity PA to significantly improve physical fitness, but only high intensity PA improved physiological outcomes such as systolic blood pressure. There appears to also be a favorable indication that, compared to low intensity, more vigorous PA is related to both achievement and elements of cognitive development. Cross-sectional findings suggest that the intensity of PA needs to be over a certain threshold to have an association with academic achievement; Ayan et al. (2014) found a positive correlation between higher achievement and more PA at high intensities in children aged 12-14. Similar findings were identified in younger children, where those who partook in vigorous PA scored higher on measures of school readiness and self-regulation (Kybartas et al., 2021).

Acute PA intensity has also been directly linked to on-task behaviour. The cognitive-energetic model (Unsworth \& Robinson, 2020) poses that a physical state of activation after PA-and in particular when this is coupled with feelings of positivity-has a beneficial impact on cognitive performance and behaviours. The percentage of PA time spent at MVPA has been positively associated with the percentage of time-on-task during subsequent instructional time (Szabo-Reed et al., 2017). And, in another investigation of on-task behaviour, Grieco et al. (2016) found that behavioural engagement increased far more after moderate-to-vigorous $(E S=1.22)$ than low-to-moderate intensity $(E S=0.43)$ PA breaks.

However, in their meta-analysis, Sember et al. (2020) noted that a limitation in many studies investigating classroom PA interventions is that the intensity of the physical activity implemented is not reported. In fact, they suggest that PA improves academic achievement not only through increased frequency of PA, but also the intensity. Therefore, in the present study it was decided to measure PA both objectively and subjectively, using accelorometer watches and children's selfreports, respectively. A complication in vigorous PA promotion in school settings is that, for many, objective PA intensity recording is not practical without the involvement of research teams and the practicality and cost of measuring equipment. And, as interventions need to be not only effective, but applicable at a larger scale (van Sluijs et al., 2021), this study investigated objective and subjective PA intensity alongside each other. Therefore, it was important for this research to incorporate subjective PA in order to establish whether children's subjective perceptions of exertion are comparable to objectively measured ratings in terms of their relationship to subsequent task-related behaviour. Subjective ratings of intensity can easily be obtained by teachers during PE lessons or PA breaks to monitor PA intensity, making them a vital aspect of schoolbased PA research.

\subsubsection{Physical Activity Complexity}

In recent years, complex, or cognitively engaging, and PA is increasingly being investigated. It is assumed that during PA, the overlapping brain regions for motor skills and cognitive functions vital to learning and attention are stimulated, which facilitates subsequent learning activity (Steinlin, 2007). Recent studies have concluded that a combination of MVPA and cognitively engagement in chronic PA is most beneficial to academic performance (Egger et al., 2019; de Bruijn et al., 2020) and executive functions (Ishihara et al., 2017), although not all studies found cognitive engagement to enhance the effects of PA interventions. For example, Meijer et al. (2021) found that the MVPA content of the intervention predicted cognitive outcomes beyond the element of cognitive engagement during PA. The acute effects of cognitively engaging PA are equally unclear, with Egger et al. (2018) reporting a deterioration in shifting performance in children directly after complex PA, whilst Benzing et al. (2016) found that cognitive flexibility of young male adolescents was improved after cognitively engaging PA. Finally, no main effect of complexity on subsequent on-task behaviour was found in the current sample in previously published results (Heemskerk et al., 2019).

\subsection{Individual Characteristics}

This study investigated the role of individual characteristics in the relationship between acute PA and task-behaviour. Characteristics included in the analyses are BMI, sex, habitual PA participation, and baseline task-related behaviour in the classroom.

\subsubsection{Baseline Task-Behaviour}

Children who spent less than $50 \%$ of instructional time on-task in the study by Mahar et al. (2006) responded far more strongly to PA breaks and improved their on-task behaviour with an effect size of $d=2.2$. Although it may seem obvious that those who start at a lower baseline have more room for improvement, and thus may respond more strongly to interventions, this still has strong educational implications. When students are off-task, they not only affect their own learning potential, but also that of those around them (Godwin \& Fisher, 2011). This is particularly the case when the off-task behaviour is active, which can disrupt whole classrooms. This study specifically investigated how PA influences those students who have the lowest levels of on-task behaviour, as well as those with the highest levels of both passive and active off-task behaviours.

\subsubsection{Sex}

Although childhood inactivity is a problem in both boys and girls (Inchley et al., 2020), girls have been found to be between 5 and $30 \%$ less active than boys (Jurakić \& Pedišić, 2013) and to benefit less from PA interventions in the school setting (Owen et al., 2017; van Sluijs et al., 2021). Individual studies have found differences in effects based on sex; Ma et al. (2014) reported favourable results for boys; although passive off-task behaviour was reduced in both sexes, and only boys reduced their active offtask behaviour. However, Wilson et al. (2016), in an all-boys 
sample, found no results of PA breaks outside of the classroom. And Grieco et al. (2016) found that changes in on-task behaviour after PA were unrelated to sex, fitness level, and or BMI.

\subsubsection{Habitual Physical Activity}

Participation in leisure time PA has been positively linked to academic outcomes in children (Erickson et al., 2015). Moreover, Syväoja et al. (2018) found that aerobic fitness-an outcome of regular exercise-positively mediated the relationship between PA and academic achievement in 9-15 year-olds. These studies did not, however, assess children's response to acute PA in light of their regular PA or level of fitness. Jäger et al. (2015) did find that the acute effects of a PA intervention session on executive functioning were significant only in higher-fit participants. This result related to updating performance, not inhibition or working memory, and no measure of behavioural engagement was involved in this study (Jäger et al., 2015).

\subsubsection{BMI}

Results on the role of BMI in the effect of PA on task-behaviour are sparse. Often, overweight and obese samples are investigated in isolation, or BMI is used as a control variable rather than a predictor. In a systematic review of $\mathrm{PA}$ interventions for overweight and obese youth, Martin et al. (2018) found no evidence of increased inhibition control after PA. This is in line with the results from a study by Mora-Gonzalez et al. (2019), who found no association of chronic MVPA or sedentary time with EF in an overweight and obese sample. In contrast to these results, Sun et al. (2021) report a standardised mean difference of SMD $=0.30$ [95\% CI 0.002-0.600] for chronic PA on core EF in studies of obese and overweight children. This is somewhat greater than the effect found by Álvarez-Bueno et al. (2017) in a meta-analysis including studies of both healthy- and overweight children (ES 0.20, [95\% CI 0.10-0.30]). Moreover, for the domain of inhibition and selective attention, they carried out a subgroup analysis based on weight status. They report an effect size for the whole sample of ES $=0.26$ [95\% CI 0.10-0.41], and an overweight and obese subgroup effect size of ES $=-0.02$ [95\% CI $-0.22-0.17$ ], suggesting a smaller effect of PA on inhibition and selective attention in overweight and obese children, compared to healthy-weight children (Álvarez-Bueno et al., 2017). Finally, in a review of studies on the effects of chronic PA and weight-status on cognitive performance, Chang et al. (2017) concluded that in studies applying models where the effects of chronic PA were moderated by BMI, results have been inconclusive. Worse effects for those with higher BMI were reported in studies with samples of morbidly obese adults, whilst a mixture of positive and null results were found in studies with children (Chang et al., 2017).

Results from studies of acute PA are also mixed. In a laboratory-based study, Vazou and Smiley-Oyen (2014) found that PA prevented the worsening of reaction times on a standard flanker task (a measure of inhibition) only in overweight children. Reaction times of healthy-weight children remained stable after both the physically active and seated maths conditions, whereas overweight children performed worse after the seated, but not the active maths task (Vazou \& Smiley-Oyen, 2014), suggesting that BMI positively moderates the effect of PA on cognition. Yet, in a
TABLE 1 | Sample descriptives.

\begin{tabular}{llcccccc}
\hline Variable & Categories & $\boldsymbol{n}$ & $\boldsymbol{M} / \%$ & SD & Min & Max & $\boldsymbol{n}_{\boldsymbol{t i}}{ }^{\mathbf{1}}$ \\
\hline Age (years) & & 76 & 9.25 & 0.66 & 7.90 & 10.44 & \\
BMI $^{2}$ & & 76 & 0.60 & 1.27 & -1.55 & 3.71 & \\
Observations & & 76 & 49.7 & 1.2 & 40 & 50 & 38,933 \\
Self-reports & Classroom & 76 & 29.7 & 7.1 & 2 & 36 & 2,259 \\
& PE & 76 & 5.5 & 0.9 & 1 & 6 & 418 \\
Sex & Girl & 46 & $60.5 \%$ & & & & \\
Handedness & Boy & 30 & $39.5 \%$ & & & & \\
& Left & 10 & $13.2 \%$ & & & & \\
Year group & Right & 66 & $86.8 \%$ & & & & \\
& Y3 & 12 & $15.8 \%$ & & & & \\
\multirow{2}{*}{ Attainment } & Y4 & 39 & $51.3 \%$ & & & & \\
& Y5 & 25 & $32.9 \%$ & & & & \\
& below ARE & 12 & $15.8 \%$ & & & & \\
Active child $^{4}$ & at ARE & 35 & $46.1 \%$ & & & & \\
& above ARE & 29 & $38.2 \%$ & & & & \\
& yes & 35 & $58.3 \%$ & & & & \\
& no & 25 & $41.7 \%$ & & & &
\end{tabular}

Note: ${ }^{1}$ Total number of items, nested in weeks, nested in participants. ${ }^{2}$ BMI z-score for age and sex, based on WHO guidelines (de Onis et al., 2007). ${ }^{3}$ ARE = Age-Related Expectation for academic achievement. ${ }^{4}$ Active child $=$ achieved government recommendation for physical activity (Chief Medical Officers, 2019) on at least $75 \%$ of recorded study days.

classroom-based study with a sample of both overweight and healthy-weight children, and specific to on-task behaviour, Grieco et al. (2016) found that the effects of PA in children aged 7 to 9 did not differ based on BMI. Moreover, Grieco et al. (2016) report that, although on-task behaviour decreased more greatly during inactive classroom lessons in children with higher BMI, PA intensity did not interact with BMI.

\section{MATERIALS AND METHODS}

\subsection{Sample and Procedure}

The study obtained ethical approval from the Departmental Research Ethics Committee at the University of Oxford Department of Education. A total sample of 108 children from grades 3-5 of four primary schools in Oxfordshire, South-East England were recruited to be part of the study. Sufficient data for analysis was available from 76 children ( $M$ age $=9.3$ years, $S D=$ 0.7 years; 46 females), including valid behaviour observations and objective and subjective PA records. Written consent was obtained from parents allowing their children to participate. Prior to commencing the study, all child participants also provided written assent and their weight and height were recorded using digital scales (Salter, model 9018 SSV3R) and measuring tape. This allowed for BMI z-scores to be calculated for each child in accordance with WHO parameters for age- and sexadjusted BMI $(M=0.60$, range $=-1.55-3.71)$. To measure objective PA, child participants wore GENEActiv accelerometers (Activeinsights, 2012) on the wrist of their non-dominant hand. The research team fitted the accelerometers on the children prior to them leaving school on the day before the intervention was to take place, so that children wore the accelerometers for $24 \mathrm{~h}$. Teachers rated 
participants' level of academic achievement on a 3-point scale: below, at, or above age-related expectation (ARE). As on-task behaviour is considered a prerequisite for positive academic outcomes (Fredricks et al., 2004), we used these teacher ratings to control for academic achievement level in all analyses. See Table $\mathbf{1}$ for a full overview of the sample descriptives.

The design of the PE lesson intervention followed a 3 (intensity: low, medium, or high) by 2 (complexity: low or high) within-person design, with the order of lessons in each classroom being randomised. For a more detailed description of every individual PE lesson please consult previous publication (Heemskerk et al., 2019). Twelve children were observed during "business as usual" classroom lessons pre- and post-the PE intervention lesson, by two trained researchers using a momentary time sampling protocol. Some transition time occurred as children returned to the classroom after the PE lessons. Between classes, the process for transition to the classroom was the same (plenary, questionnaire, lining up, and walking back to the classroom without getting changed back into uniform). Within classes, the transition time was similar across the six conditions, and as the distance traveled from PE to the classroom was the same each week. Both the children and teachers were encouraged to resume the regular lesson post-PE as quickly and seamlessly as possible. Observations resumed when both observers agreed that the teacher was initiating the classroom lesson. In order to maintain ecological validity, this process was not timed or controlled across classrooms. It is likely and expected that some children would re-focus more quickly and easily than others, and this is some of the naturally occurring variance we aimed to capture an investigate.

In cases where more than twelve children had parental consent, the class teacher chose the twelve students to take part in the study to ensure an even distribution of sex and academic achievement. In addition, children rated their learning experiences at the start, middle and end of each observation period, and at the end of the PE lesson, and using a purpose-built app.

\subsection{Physical Activity}

The six intervention lessons were designed in accordance with the United Kingdom National Curriculum for PE requirements, which includes athletics, health-related fitness, games and dance. No specialist equipment was required for any of the lessons, and the minimal equipment used for some lessons was already available in the schools. Two PE lessons each were designed to ensure the participants reached adequate levels of VPA, MPA, and LPA. The intensity level was manipulated by adjusting the pace of movements (walking, jogging, or sprinting), the amount of locomotion required (stationary drills or moving) and the amount of vertical movement (jumping). For example, in one low intensity lesson, children worked on flexibility and flexibility testing, whereas in the high intensity lesson they performed sprinting races and sprint relays. At each of the three intensity levels, one of two lessons required simple and automated movements, while the other challenged participants with more complex movements, external pacing and/or rapidly changing environments the children needed to adapt to. For example, in the low complexity high intensity lesson, the participants sprinted in straight lines, which is an automated movement for children. In the corresponding high complexity lesson, they did aerobics. This involved music-directed pacing and the complex combination of arm and leg movements.

Children's activity levels were tracked using the accelerometer devices during the PE lessons, as well as their free-living PA, which was recorded for up to $24 \mathrm{~h}$ leading up to the intervention lesson. The activity recordings of the PE lessons were removed from the free-living PA data for analysis purposes. The free-living data was used to ascertain whether children met the United Kingdom government's $60 \mathrm{~min}$ of daily MVPA guideline (Chief Medical Officers, 2019).

\subsection{Self-Reports}

Children reported their learning experiences (task enjoyment, perceived task difficulty, tiredness, positive, and negative affect) on a 6-point Likert scale, three times during their regular classroom lesson before and after the $\mathrm{PE}$ intervention, and once after the PE lesson. As this was a repeated measure, the questionnaire was kept short and concise and took around $30 \mathrm{~s}$ to complete. Affect was measured with eight items (four positive, four negative), using the question "How do you feel right now?". In the classroom, enjoyment, difficulty, and tiredness were measured by one item each ("Do you like doing this task?", "How hard is this task?", and "Are you tired?", respectively). Affect and tiredness in the classroom were not analysed in the context of the results presented here. Classroom task enjoyment and perceived difficulty were used as control variables, as the lessons before and after PE were on different subjects. At the end of PE lessons, with one item each, participants rated how much they had enjoyed the PE lesson and provided their rate of perceived exertion (RPE) as a subjective measure of the PE lesson intensity, using the same 6point Likert scale. A 7-step version of Borg's RPE scale has previously been validated for use with children (Groslambert et al., 2001). We decided to keep to our 6-point scale for conformity with the other questionnaire items. Answer options were simplified with the use of emoji's (Rane, 2017) and were given as a rating from zero to five stars.

\subsection{Behaviour Observations}

Two trained researchers carried out the observations; their interrater reliability was good (Cohen's Kappa $=0.8$ ). The observation protocol required the observers to record each participants' behaviour every $30 \mathrm{~s}$ for $25 \mathrm{~min}$. Each observation was rated as either "on-task" if the child showed goal-directed behaviours and was completing a task as set by the teacher, "off-task passive" if the child did not demonstrate any goal-directed behaviours but was instead inactive (e.g., staring or daydreaming), "off-task active" if no goal-directed behaviours were observed and the child was active (e.g., non task-related talking, fidgeting in their seat or out of their seat moving), or "other" if the observed behaviour did not fit one of the three aforementioned categories, 
or the child was out of sight (e.g., left the classroom, another person obstructing the view of the observer). As it has previously been found that on-task behaviour depends on and varies according to the type of instructional activity the children are engaging in (Godwin et al., 2013; Heemskerk and Malmberg, 2020), the researchers recorded the type of task that was set by the teacher at each observation (teacher-led whole class instruction, teacher one-to-one support, independent work, partner work, small group work, test, and or other).

\subsection{Analytic Strategy}

\subsubsection{Variable Creation}

The accelerometery data was processed with GGIR in R (Migueles et al., 2019). We aggregated data in 1-s epochs, and extracted the duration participants were sedentary, and engaged in light, moderate, and vigorous activity for sections of the $24 \mathrm{~h}$ period during which the device was worn: "after school", "night-time" (8 pm until 7 am), "before school", "morning school”, "lunchtime”, "afternoon before PE", "PE", and "afternoon after PE". Cut-points for sedentary, moderate, and vigorous PA from Hildebrand et al. (2014) for wrist-worn GENEActiv monitors were applied. Files with a minimum of $8 \mathrm{~h}$ of valid data during the daytime ( $7 \mathrm{am}$ until $8 \mathrm{pm}$ ) were included for analyses of free-living PA. For PE lessons, at least $30 \mathrm{~min}$ of valid data was required for inclusion. PA during $\mathrm{PE}$ lessons was excluded from free-living PA variables due to the intervention manipulation having a potential impact on the values. Free-living data was used for the calculation of the "active child" variable, reflecting if a child met the government guidelines for MVPA (Chief Medical Officers, 2019) on a regular basis ( $\geq 75 \%$ of recorded days). This applied to $58.3 \%$ of those with sufficient PA data to calculate this variable ( 35 of 60 participants). For analyses of objectively achieved PA during PE, the duration of MVPA was coded into quintiles and used as a categorical predictor (quintile 1: $<14 \%$, quintile 2: $14-21 \%$, quintile $3:>21$ and $<27.2 \%$, quintile 4 : 27.2 and $<33.5 \%$, quintile $5: \geq 33.5 \%$ ).

Observations where behaviour and task were concurrently coded "other" (indicating the child was absent or obscured from view) were deleted, and only lessons with a minimum of 40 valid observations were included in the analyses. Classroom behaviour data was then recoded into three binary variables (on-task, passive off-task, and active off-task). Trait taskbehaviour was coded into three binary indicators. For each participant, their mean proportion of on-task, passive off-task, and active off-task behaviour across all classroom lessons before PE only was calculated. Children who were in the bottom $33 \%$ of the sample for on-task behaviour (on-task < $66 \%$ ) or the top $33 \%$ for passive (passive off-task $>11.5 \%$ ) or active off-task (active off-task $>15 \%$ ) behaviour were coded "low on-task", "high passive off-task", and "high active offtask" respectively.

BMI values were coded into $\mathrm{z}$-scores for sex and age using the WHO guidelines (de Onis et al., 2007). For use in the binary model, a categorical variable was created on the basis of the $\mathrm{z}$-scores, using cut-points for underweight $(\mathrm{z}<-1)$ (Cole et al., $2007)$, overweight $(z>1.04)$, and obese $(z>1.64)$ (Vanderwall et al., 2018). The "healthy weight" category $(z \geq-1$ and $\leq 1.04)$ was used as the comparison category.

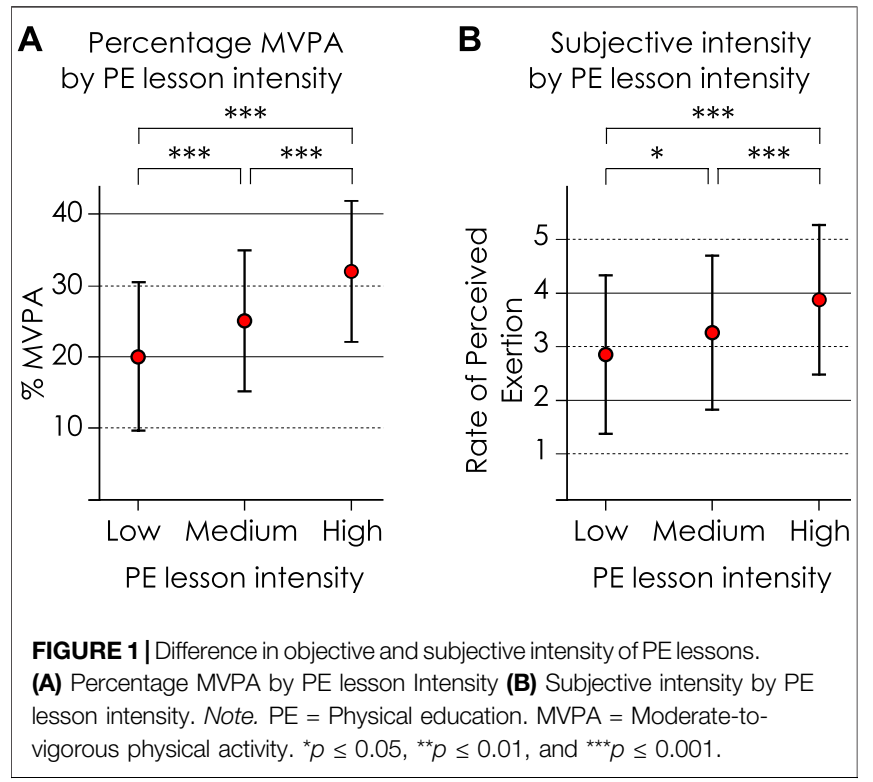

\subsubsection{Manipulation Checks}

First, we used SPSS 27 (IBM Corporation, 2020) to carry out $t$-tests to investigate if the objective or subjective intensity of the PE lessons differed between low and high complexity PE lessons. Objective PA intensity (\% of PE lesson time spent at MVPA) did differ between low and high complexity lessons, with more MVPA recorded during high complexity lessons $\left(M_{\text {low }}=8.31, M_{\text {high }}=9.40\right.$, and $\left.t_{(326)}=-11.02, p<0.001\right)$. As high complexity lessons involved more object manipulation activities, it was expected that the wrist-worn accelerometers may pick up additional activity during high complexity lessons. We therefor also analysed the subjective intensity (rate of perceived exertion) in relation to lesson complexity. This was not significantly different between low and high complexity lessons $\left(M_{\text {low }}=3.34, M_{\text {high }}=3.14, t_{(402)}=1.36\right.$, and $p=0.175$ ).

Next, we carried out a one-way ANOVA to investigate if the percentage of lesson time spent in MVPA differed between low, medium, and high intensity lessons. This was the case $\left(F_{(2,456)}=\right.$ $68.79, p<0.001$ ), and post-hoc tests revealed that during low intensity lessons, participants achieved significantly less MVPA than during medium intensity lessons, and during medium intensity lessons they achieved significantly less MVPA than during high intensity lessons (see Figure 1A). The same pattern was found for subjective intensity level, which also differed significantly between the three PE lesson intensities (Welch's $\left.F_{(2,375.34)}=24.92, p<0.001\right)$, (see Figure 1B).

\subsubsection{Analytic Models}

We used binary three-level models (time points, nested in weeks, nested in participants) with random intercepts and slopes in MLwiN (Charlton et al., 2019) to analyse the effect of PA on classroom behaviour. The intra-class correlations for each behaviour were calculated at the week-level and ID- 
TABLE 2 | Response of on-task behaviour to objective and subjective PA.

\begin{tabular}{|c|c|c|c|c|c|c|}
\hline \multirow[t]{2}{*}{ Variable } & \multicolumn{6}{|c|}{ On-task behaviour } \\
\hline & Probability pre-PE & Probability post-PE & $x^{2}$ & $p$-value & Odds ratio & $d$ \\
\hline Overall & 0.647 & 0.709 & & & 1.33 & 0.16 \\
\hline Medium intensity lesson & 0.629 & 0.683 & 3.65 & & 1.27 & 0.13 \\
\hline High intensity lesson & 0.651 & 0.735 & 10.08 & *** & 1.49 & 0.22 \\
\hline MVPA $^{1}$ quintile 1 & 0.647 & 0.703 & 2.89 & & 1.30 & 0.14 \\
\hline MVPA quintile 4 & 0.607 & 0.701 & 6.03 & * & 1.52 & 0.23 \\
\hline MVPA quintile 5 & 0.609 & 0.747 & 12.26 & 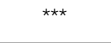 & 1.90 & 0.35 \\
\hline $\mathrm{RPE}^{2} 1$ & 0.658 & 0.722 & 3.40 & & 1.35 & 0.17 \\
\hline RPE 2 & 0.669 & 0.687 & 0.22 & & 1.09 & 0.05 \\
\hline RPE 3 & 0.633 & 0.704 & 3.90 & * & 1.37 & 0.18 \\
\hline
\end{tabular}

Note: ${ }^{1}$ MVPA = Moderate-to-Vigorous Physical Activity during physical education, coded into quintiles. ${ }^{2}$ RPE = Rate of Perceived Exertion, rated from 1 (not at all tiring) to 5 (extremely tiring). ${ }^{*} \mathrm{p} \leq 0.05,{ }^{* *} \mathrm{p} \leq 0.01$, and ${ }^{* * *} \mathrm{p} \leq 0.001$. Effect sizes indicating a small ( $\left.\mathrm{d} \geq 0.2\right)$, medium ( $\left.\mathrm{d} \geq 0.5\right)$, or large ( $\mathrm{d} \geq 0.8$ ) effect are highlighted in bold font.

level. For on-task behaviour, greater variance between weeks than between participants was found $\left(I C C_{\text {weeks }}=0.080\right.$, $I C C_{\text {participants }}=0.047$ ), whereas for off-task behaviour the variance did not greatly differ, and was slightly greater between participants than between weeks (active off-task: $I C C_{\text {weeks }}=0.096, I C C_{\text {participants }}=0.099 ;$ passive off-task: $I C C_{\text {weeks }}=0.090$, and $\left.I C C_{\text {participants }}=0.094\right)$.

We answered the first research question by entering PA into the model in the following three ways; in the first model, the intensity level of the six intervention conditions (low, medium, or high) was used as the predictor. In the second model, the percentage of time spent in MVPA as quintiles was entered. In model three, participants' subjective PA intensity during PE was used. To answer our second research question, the models using MVPA for objective PA and RPE for subjective PA were computed twice more each, once with active off-task and once with passive off-task behaviour as the outcome variable. Finally, to investigate the effect of individual differences and answer our third research question, interaction effects of BMI category, sex, habitual PA participation (active child), and mean on-task and offtask behaviours in pre-PE lessons were entered into the models. For the analytical models we present $c h i^{2}$ values derived from Wald tests in MLwiN for the comparison of pre- and post-PE lessons, along with associated $p$-values (cutpoint for significance set at $p \leq 0.05)$. Effect sizes are presented as odds ratios (OR) and Cohen's $d$ (small effect $d \geq 0.2$, medium effect $d \geq 0.5$, and large effect $d \geq 0.8$ ). The reported predicted probabilities for on- and off-task behaviours are calculated from the logit coefficients provided by MLwiN, using the equation "probability = $(\exp (\operatorname{logit}) /(1+\exp (\operatorname{logit})))$ ". All models were controlled for age, sex, BMI, achievement level, task enjoyment, perceived task difficulty, and instructional activity. Full model specifications are available from the corresponding author upon request.

\section{RESULTS}

\subsection{RQ1: Is There a Difference Between the Effects of Subjective and Objective Measures of PA Intensity?}

Participants were on-task on average $71.6 \%$ of the observed pre-PE lessons, and $77.0 \%$ of the post-PE lessons. Lessons that were intended to be high-intensity had a small positive effect on on-task behaviour $\left(\chi^{2}=10.08, p<0.001\right.$, and $\left.d=0.22\right)$. Based on recorded MVPA, lessons with more than $27.2 \%$ MVPA (4th and 5th quintile) had a small positive effect on subsequent on-task behaviour $\left(\chi^{2}=6.03, p<0.05, d=0.23\right.$ and $\chi^{2}=12.26, p<0.001, d=0.35$, respectively). This did not directly translate to participants' RPE; only lessons rated 4 out of 5 had a small significant positive effect $\left(\chi^{2}=6.62, p<0.01\right.$, and $d=0.25)$. When RPE was 3 out of 5 , subsequent on-task behaviour was significantly greater $\left(\chi^{2}=3.90, p<0.05\right)$, but this effect was very small $(d=0.18)$ see Table 2; Figure 2 .

\subsection{RQ2: Is There a Difference Between the Effects of PA on Active and Passive Off-Task Behaviour?}

Across the afternoon, active off-task behaviour was more prevalent than passive (pre: $\mathrm{M}_{\text {passive }}=10.5 \%, \mathrm{M}_{\text {active }}=$ $14.4 \%$; post: $\left.\mathrm{M}_{\text {passive }}=7.5 \%, \mathrm{M}_{\text {active }}=13.3 \%\right)$. Active offtask behaviour was significantly reduced only after $\mathrm{PE}$ lessons with high MVPA content (quintile 5, $\chi^{2}=5.46, p<$ 0.05 , and $d=-0.27$ ). No significant effects of subjective intensity ratings on active off-task were found (see Table 3; Figure 3, dark grey bars). Passive off-task behaviour was lower after several different PE lessons of low and moderate-but not high-intensity. When intensity was measured objectively, lessons with MVPA in quintile 2 had a small negative effect $\left(\chi^{2}=7.98, p<0.01\right.$, and $\left.\mathrm{d}=-0.3\right)$. When PA was measured 


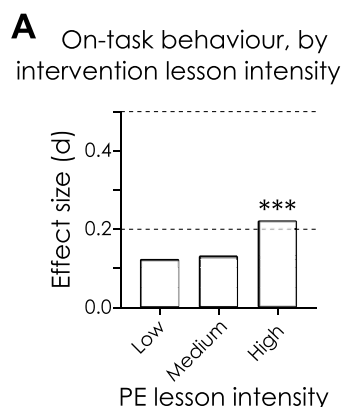

B On-task behaviour, by
MVPA quintile

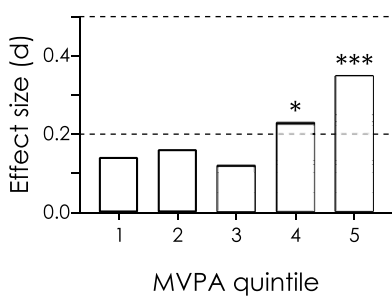

C On-task behaviour, by RPE

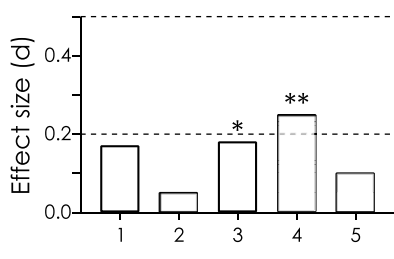

Rate of perceived exertion

FIGURE 2 | Change in on-task behaviour in response to physical activity intensity. (A) On-task behaviour, by intervention lesson intensity (B) On-task behaviour, by MVPA quintile (C) On-task behaviour, RPE. Note. MVPA = Moderate-to-vigorous physical activity. Small $(d=0.2)$, medium $(d=0.5)$, and large $(d=0.8)$ effect sizes are indicated by the dashed grid lines. ${ }^{\star} p \leq 0.05,{ }^{\star \star} p \leq 0.01$, and ${ }^{\star \star \star} p \leq 0.001$.

TABLE 3 | Response of active off-task behaviour to objective and subjective PA

Variable

Active off-task behaviour

\begin{tabular}{|c|c|c|c|c|c|c|}
\hline & Probability pre-PE & Probability post-PE & $\chi^{2}$ & $p$-value & Odds ratio & $d$ \\
\hline Overall & 0.193 & 0.177 & & & 0.89 & -0.06 \\
\hline MVPA $^{1}$ quintile 1 & 0.175 & 0.156 & 0.62 & & 0.87 & -0.08 \\
\hline MVPA quintile 2 & 0.180 & 0.159 & 0.61 & & 0.86 & -0.08 \\
\hline MVPA quintile 3 & 0.193 & 0.206 & 0.20 & & 1.09 & 0.05 \\
\hline MVPA quintile 4 & 0.202 & 0.192 & 0.11 & & 0.94 & -0.04 \\
\hline MVPA quintile 5 & 0.237 & 0.160 & 5.46 & * & 0.61 & -0.27 \\
\hline $\mathrm{RPE}^{2} 1$ & 0.190 & 0.177 & 0.23 & & 0.92 & -0.05 \\
\hline RPE 2 & 0.168 & 0.189 & 0.50 & & 1.16 & 0.08 \\
\hline RPE 3 & 0.214 & 0.196 & 0.35 & & 0.90 & -0.06 \\
\hline RPE 4 & 0.188 & 0.173 & 0.26 & & 0.90 & -0.06 \\
\hline RPE 5 & 0.219 & 0.197 & 0.58 & & 0.87 & -0.07 \\
\hline
\end{tabular}

Note: ${ }^{1}$ MVPA = Moderate-to-Vigorous Physical Activity during physical education, coded into quintiles. ${ }^{2}$ RPE = Rate of Perceived Exertion, rated from 1 (not at all tiring) to 5 (extremely tiring). ${ }^{*} \mathrm{p} \leq 0.05,{ }^{* *} \mathrm{p} \leq 0.01$, and ${ }^{* *} \mathrm{p} \leq 0.001$. Effect sizes indicating a small ( $\left.\mathrm{d} \geq 0.2\right)$, medium ( $\left.\mathrm{d} \geq 0.5\right)$, or large ( $\left.\mathrm{d} \geq 0.8\right)$ effect are highlighted in bold font.

A Off-task behaviour, by MVPA quintile

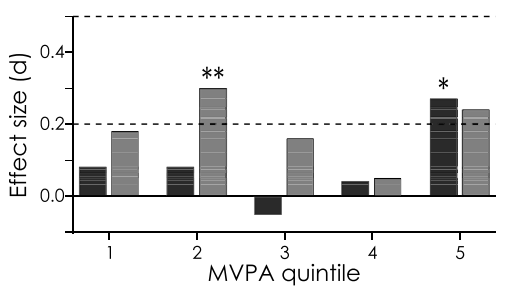

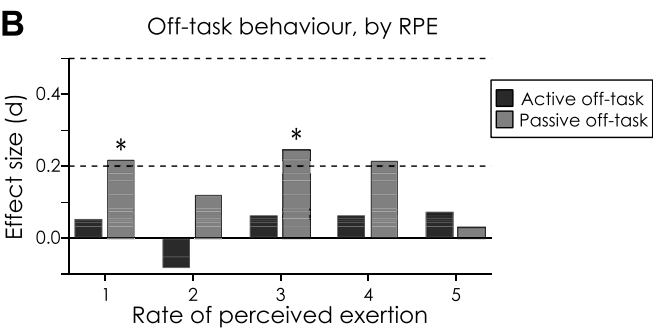

FIGURE 3 | Change in off-task behaviour in response to physical activity intensity. (A) Off task behaviour, MVPA quintile (B) Off-task behaviour, by RPE. Note. MVPA = Moderate-to-vigorous physical activity. Effect sizes have been transformed so that positive numbers reflect favourable effects (less off-task behaviour). Small $(d=0.2)$, medium $(d=0.5)$, and large $(d=0.8)$ effect sizes are indicated by the dashed grid lines. ${ }^{*} p \leq 0.05,{ }^{* \star} p \leq 0.01$, and ${ }^{\star * *} p \leq 0.001$.

subjectively, low $(\mathrm{RPE}=1)$ as well as moderate $(\mathrm{RPE}=3)$ intensity lessons had a small negative effect on passive off-task behaviour $\left(\chi^{2}=4.27, p<0.05\right.$, and $d=-0.22 ; \chi^{2}=5.45, p<0.05$, and $d=-0.25$, respectively). Although lessons in MVPA quintile 5 and rated as $\mathrm{RPE}=4$ did achieve small effect sizes, these results were not significant (see Figure 3, light grey bars and Table 4).

\subsection{RQ3: Are There Specific Sub-Samples of Participants Who Respond More or Less Favourably to PA?}

\subsubsection{Trait On-Task Behaviour}

Participants who were the least on-task before PE benefited from all PE lessons, regardless if subjective or objective measures were 
TABLE 4 | Response of passive off-task behaviour to objective and subjective PA.

\begin{tabular}{|c|c|c|c|c|c|c|}
\hline \multirow[t]{2}{*}{ Variable } & \multicolumn{6}{|c|}{ Passive off-task behaviour } \\
\hline & Probability pre-PE & Probability post-PE & $\chi^{2}$ & $p$-value & Odds ratio & $d$ \\
\hline Overall & 0.146 & 0.115 & & & 0.76 & -0.15 \\
\hline MVPA $^{1}$ quintile 1 & 0.184 & 0.140 & 3.67 & & 0.72 & -0.18 \\
\hline MVPA quintile 2 & 0.185 & 0.117 & 7.98 & ** & 0.58 & -0.30 \\
\hline MVPA quintile 3 & 0.184 & 0.145 & 2.34 & & 0.75 & -0.16 \\
\hline MVPA quintile 4 & 0.137 & 0.126 & 0.25 & & 0.91 & -0.05 \\
\hline MVPA quintile 5 & 0.148 & 0.102 & 3.37 & & 0.65 & -0.24 \\
\hline $\mathrm{RPE}^{2} 1$ & 0.156 & 0.111 & 4.27 & * & 0.67 & -0.22 \\
\hline RPE 2 & 0.139 & 0.114 & 1.05 & & 0.80 & -0.12 \\
\hline RPE 3 & 0.152 & 0.103 & 5.45 & * & 0.64 & -0.25 \\
\hline RPE 4 & 0.160 & 0.113 & 3.56 & & 0.67 & -0.22 \\
\hline RPE 5 & 0.140 & 0.134 & 0.08 & & 0.95 & -0.03 \\
\hline
\end{tabular}

Note: ${ }^{1}$ MVPA = Moderate-to-Vigorous Physical Activity during physical education, coded into quintiles. ${ }^{2}$ RPE = Rate of Perceived Exertion, rated from 1 (not at all tiring) to 5 (extremely tiring). ${ }^{*} \mathrm{p} \leq 0.05,{ }^{* *} \mathrm{p} \leq 0.01$, and ${ }^{* * *} \mathrm{p} \leq 0.001$. Effect sizes indicating a small ( $\left.\mathrm{d} \geq 0.2\right)$, medium ( $\mathrm{d} \geq 0.5$ ), or large ( $\mathrm{d} \geq 0.8$ ) effect are highlighted in bold font.

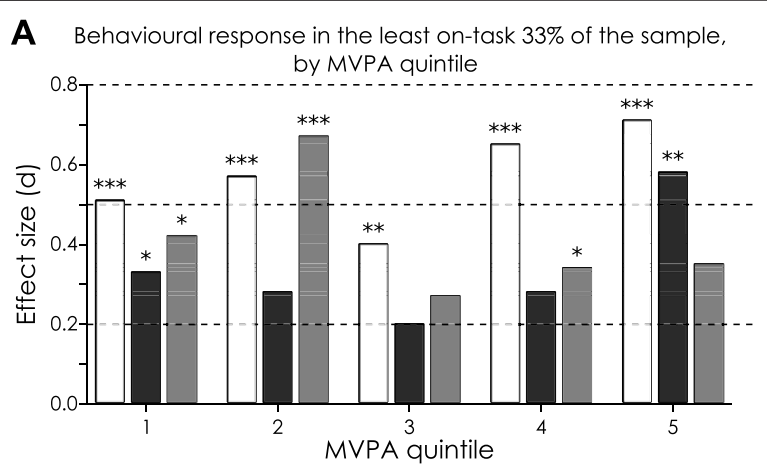

B Behavioural response in the least on-task $33 \%$ of the sample, by RPE

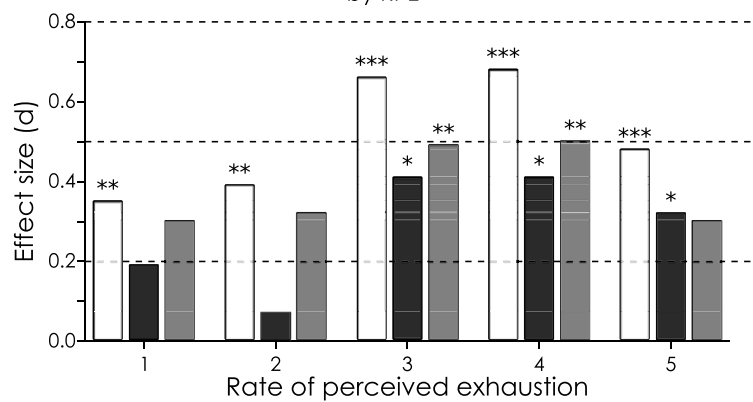

FIGURE 4 | Change in behaviour in response to objective and subjective measures of physical activity intensity in the least on-task participants. (A) Behavioural response in the least on-task 33\% of the sample, MVPA quintile (B) Behavioural response in the least on-task $33 \%$ of the sample, by RPE. Note. MVPA = Moderate-tovigorous physical activity. Effect sizes have been transformed so that positive numbers reflect favourable effects (more on-task behaviour, less off-task behaviour). Small $(d=0.2)$, medium $(d=0.5)$, and large $(d=0.8)$ effect sizes are indicated by the dashed grid lines. ${ }^{\star} p \leq 0.05,{ }^{* *} p \leq 0.01$, and ${ }^{* \star *} p \leq 0.001$.

used. Full results can be found in Table 5. Every PE lesson had a positive effect on their subsequent on-task behaviour (objective: $d=0.40-0.71$, subjective: $d=0.35-0.68$ ). Children with low trait levels of on-task behaviour reduced their passive off-task behaviours after PE lessons of sub-maximal intensity-both for subjectively and objectively measured PA the maximum intensity category failed to reach significance (MVPA quintile 5: $\chi^{2}=3.60, p>0.05$; RPE 5: $\chi^{2}=3.70, p>0.05$ ). Active off-task behaviour was lower in this subgroup after subjective intensities of 3 and above $\left(\chi^{2}=4.51-5.81, p<0.05\right.$, and $d=-0.32$ to -0.41$)$. Objectively measured PA did not affect active off-task behaviour in a systematic way (see Figure 4). 

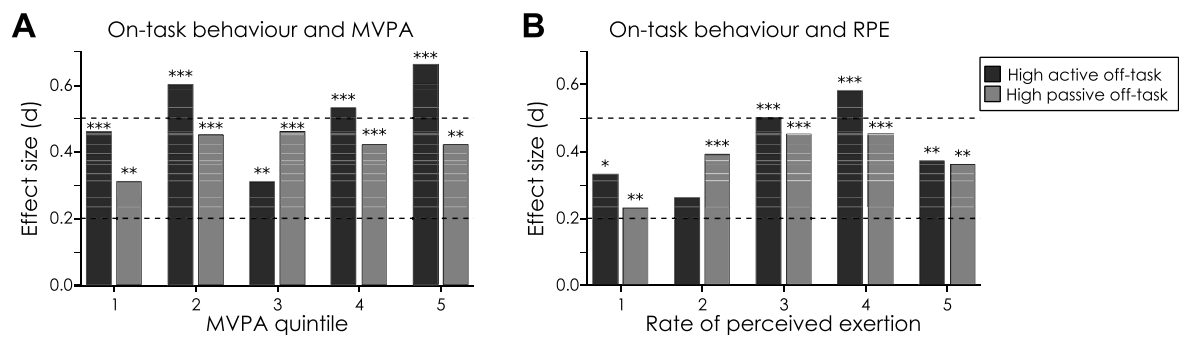

FIGURE 5 | Change in behaviour in response to objective and subjective measures of physical activity intensity in the most off-task participants. (A) On-task behaviour and MVPA (B) On-task behaviour and RPE. Note. MVPA = Moderate-to-vigorous physical activity. Effect sizes have been transformed so that positive numbers reflect favourable effects (more on-task behaviour, less off-task behaviour). Small ( $d=0.2)$, medium ( $d=0.5)$, and large ( $d=0.8$ ) effect sizes are indicated by the dashed grid lines. ${ }^{*} p \leq 0.05,{ }^{\star *} p \leq 0.01$, and ${ }^{* \star *} p \leq 0.001$.

TABLE 5 | Response of task behaviour to objective and subjective PA in the least on-task sub-sample.

\begin{tabular}{|c|c|c|c|c|c|c|c|c|c|c|c|c|}
\hline \multirow[t]{2}{*}{ Variable } & \multicolumn{4}{|c|}{ On-task behaviour } & \multicolumn{4}{|c|}{ Active off-task behaviour } & \multicolumn{4}{|c|}{ Passive off-task behaviour } \\
\hline & $x^{2}$ & $p$-value & $\begin{array}{l}\text { Odds } \\
\text { ratio }\end{array}$ & $d$ & $x^{2}$ & $p$-value & $\begin{array}{c}\text { Odds } \\
\text { ratio }\end{array}$ & $d$ & $x^{2}$ & $p$-value & $\begin{array}{l}\text { Odds } \\
\text { ratio }\end{array}$ & $d$ \\
\hline $\begin{array}{l}\text { MVPA }^{1} \\
\text { quintile } 1\end{array}$ & 16.39 & $\star \star \star ~$ & 2.54 & 0.51 & 4.31 & * & 0.55 & -0.33 & 6.62 & $\star \star$ & 0.47 & -0.42 \\
\hline $\begin{array}{l}\text { MVPA } \\
\text { quintile } 2\end{array}$ & 18.45 & $\star * * *$ & 2.81 & 0.57 & 2.98 & & 0.60 & -0.28 & 6.62 & $\star \star * *$ & 0.29 & -0.67 \\
\hline $\begin{array}{l}\text { MVPA } \\
\text { quintile } 3\end{array}$ & 10.09 & $\star * \star *$ & 2.06 & 0.40 & 1.61 & & 0.70 & -0.20 & 2.90 & & 0.61 & -0.27 \\
\hline $\begin{array}{l}\text { MVPA } \\
\text { quintile } 4\end{array}$ & 24.34 & 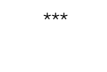 & 3.24 & 0.65 & 3.11 & & 0.60 & -0.28 & 3.97 & * & 0.54 & -0.34 \\
\hline $\begin{array}{l}\text { MVPA } \\
\text { quintile } 5\end{array}$ & 25.04 & $\star * \star *$ & 3.62 & 0.71 & 11.42 & 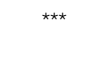 & 0.35 & -0.58 & 3.60 & & 0.53 & -0.35 \\
\hline $\mathrm{RPE}^{2} 1$ & 7.94 & $\star \star$ & 1.90 & 0.35 & 1.45 & & 0.71 & -0.19 & 3.14 & & 0.58 & -0.30 \\
\hline RPE 2 & 6.97 & ** & 2.03 & 0.39 & 0.13 & & 0.89 & -0.07 & 2.54 & & 0.56 & -0.32 \\
\hline RPE 3 & 23.79 & $\star \star \star \star ~$ & 3.29 & 0.66 & 5.81 & * & 0.48 & -0.41 & 7.29 & $\star \star$ & 0.41 & -0.49 \\
\hline RPE 4 & 22.26 & $\star \star \star \star ~$ & 3.41 & 0.68 & 5.17 & * & 0.48 & -0.41 & 6.78 & $\star \star$ & 0.41 & -0.50 \\
\hline RPE 5 & 17.03 & 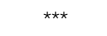 & 2.39 & 0.48 & 4.51 & * & 0.56 & -0.32 & 3.70 & & 0.58 & -0.30 \\
\hline
\end{tabular}

Note: ${ }^{1}$ MVPA = Moderate-to-Vigorous Physical Activity during physical education, coded into quintiles. ${ }^{2}$ RPE = Rate of Perceived Exertion, rated from 1 (not at all tiring) to 5 (extremely tiring). ${ }^{*} \mathrm{p} \leq 0.05,{ }^{* *} \mathrm{p} \leq 0.01$, and ${ }^{* * *} \mathrm{p} \leq 0.001$. Effect sizes indicating a small $(\mathrm{d} \geq 0.2)$, medium $(\mathrm{d} \geq 0.5)$, or large ( $\left.\mathrm{d} \geq 0.8\right)$ effect are highlighted in bold font.

TABLE 6 | Response of on-task behaviour to objective and subjective PA in the most off-task sub-samples.

\begin{tabular}{|c|c|c|c|c|c|c|c|c|}
\hline \multirow[t]{2}{*}{ Variable } & \multicolumn{4}{|c|}{ Most active off-task } & \multicolumn{4}{|c|}{ Most passive off-task } \\
\hline & $\chi^{2}$ & $p$-value & OR $^{1}$ & $d$ & $x^{2}$ & $p$-value & OR & $d$ \\
\hline MVPA $^{2}$ quintile 1 & 19.75 & 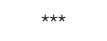 & 2.30 & 0.46 & 9.32 & $\star \star$ & 1.77 & 0.31 \\
\hline MVPA quintile 2 & 30.93 & 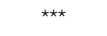 & 2.97 & 0.60 & 18.22 & 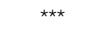 & 2.25 & 0.45 \\
\hline MVPA quintile 3 & 8.68 & $\star \star$ & 1.76 & 0.31 & 18.16 & 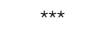 & 2.31 & 0.46 \\
\hline MVPA quintile 4 & 22.50 & 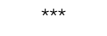 & 2.60 & 0.53 & 14.94 & 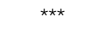 & 2.14 & 0.42 \\
\hline MVPA quintile 5 & 33.35 & 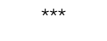 & 3.34 & 0.66 & 7.51 & $\star \star$ & 2.13 & 0.42 \\
\hline $\mathrm{RPE}^{3} 1$ & 6.28 & * & 1.82 & 0.33 & 2.64 & & 1.52 & 0.23 \\
\hline RPE 2 & 2.74 & & 1.60 & 0.26 & 6.44 & * & 2.04 & 0.39 \\
\hline RPE 3 & 12.84 & $\star \star \star ~$ & 2.49 & 0.50 & 11.29 & $\star \star$ & 2.27 & 0.45 \\
\hline RPE 4 & 16.29 & $\star \star \star$ & 2.85 & 0.58 & 9.67 & ** & 2.24 & 0.45 \\
\hline RPE 5 & 8.41 & $\star \star$ & 1.97 & 0.37 & 9.02 & $\star \star$ & 1.92 & 0.36 \\
\hline
\end{tabular}

Note: ${ }^{1}$ OR $=$ Odds Ratio. ${ }^{2}$ MVPA = Moderate-to-Vigorous Physical Activity during physical education, coded into quintiles. ${ }^{3}$ RPE $=$ Rate of Perceived Exertion, rated from 1 (not at all tiring) to 5 (extremely tiring). ${ }^{*} \mathrm{p} \leq 0.05$, ** $\mathrm{p} \leq 0.01$, and ${ }^{* * *} \mathrm{p} \leq 0.001$. Effect sizes indicating a small $(\mathrm{d} \geq 0.2)$, medium (d $\left.\geq 0.5\right)$, or large (d $\geq 0.8$ ) effect are highlighted in bold font. 
TABLE 7 | Response of task-behaviour to objective and subjective PA by sex.

\begin{tabular}{|c|c|c|c|c|c|c|c|c|c|}
\hline \multirow[t]{3}{*}{ Outcome } & \multirow[t]{3}{*}{ Variable } & \multicolumn{8}{|c|}{ Sex } \\
\hline & & \multicolumn{4}{|c|}{ Boys } & \multicolumn{4}{|c|}{ Girls } \\
\hline & & $\chi^{2}$ & $p$-value & OR $^{1}$ & $d$ & $\chi^{2}$ & $p$-value & OR & $d$ \\
\hline \multirow[t]{10}{*}{ On-task behaviour } & MVPA $^{2}$ quintile 1 & 0.08 & & 0.93 & -0.04 & 2.71 & & 1.51 & 0.23 \\
\hline & MVPA quintile 2 & 0.00 & & 1.00 & 0.00 & 3.01 & & 1.54 & 0.24 \\
\hline & MVPA quintile 3 & 0.03 & & 1.05 & 0.02 & 1.52 & & 1.36 & 0.17 \\
\hline & MVPA quintile 4 & 0.53 & & 1.22 & 0.11 & 5.33 & * & 1.85 & 0.34 \\
\hline & MVPA quintile 5 & 3.93 & * & 1.74 & 0.31 & 5.61 & * & 1.96 & 0.37 \\
\hline & $\mathrm{RPE}^{3} 1$ & 0.03 & & 1.05 & 0.02 & 4.49 & * & 1.71 & 0.30 \\
\hline & RPE 2 & 1.42 & & 1.42 & 0.19 & 0.00 & & 1.02 & 0.01 \\
\hline & RPE 3 & 1.91 & & 1.44 & 0.20 & 1.92 & & 1.39 & 0.18 \\
\hline & RPE 4 & 0.00 & & 1.01 & 0.00 & 8.80 & $\star *$ & 2.162 & 0.43 \\
\hline & RPE 5 & 0.15 & & 0.91 & -0.05 & 2.40 & & 1.45 & 0.20 \\
\hline \multirow[t]{10}{*}{ Passive off-task behaviour } & MVPA quintile 1 & 2.27 & & 0.66 & -0.23 & 0.82 & & 0.77 & -0.14 \\
\hline & MVPA quintile 2 & 1.12 & & 0.66 & -0.19 & 4.95 & * & 0.53 & -0.35 \\
\hline & MVPA quintile 3 & 0.64 & & 0.71 & -0.14 & 1.09 & & 0.74 & -0.17 \\
\hline & MVPA quintile 4 & 3.20 & & 1.72 & 0.30 & 4.31 & * & 0.52 & -0.37 \\
\hline & MVPA quintile 5 & 1.49 & * & 0.67 & -0.22 & 1.68 & & 0.64 & -0.25 \\
\hline & $\mathrm{RPE}^{3} 1$ & 1.25 & & 0.72 & -0.18 & 2.30 & & 0.62 & -0.26 \\
\hline & RPE 2 & 1.70 & & 0.61 & -0.27 & 0.23 & & 0.86 & -0.08 \\
\hline & RPE 3 & 0.83 & & 0.75 & -0.16 & 3.34 & & 0.59 & -0.29 \\
\hline & RPE 4 & 0.00 & & 1.01 & 0.01 & 4.38 & * & 0.51 & -0.37 \\
\hline & RPE 5 & 0.09 & & 1.09 & 0.05 & 0.24 & & 0.87 & -0.08 \\
\hline
\end{tabular}

Note: ${ }^{1}$ OR $=$ Odds Ratio. ${ }^{2}$ MVPA $=$ Moderate-to-Vigorous Physical Activity during physical education, coded into quintiles. ${ }^{3} R P E=$ Rate of Perceived Exertion, rated from 1 (not at all tiring) to 5 (extremely tiring). ${ }^{*} \mathrm{p} \leq 0.05,{ }^{* *} \mathrm{p} \leq 0.01,{ }^{* * *} \mathrm{p} \leq 0.001$. Effect sizes indicating a small ( $\mathrm{d} \geq 0.2$ ), medium (d $\geq 0.5$ ), or large (d $\geq 0.8$ ) effect are highlighted in bold font.

TABLE 8 | Response of task-behaviour to objective and subjective PA by regular moderate-to-vigorous physical activity.

\begin{tabular}{|c|c|c|c|c|c|c|c|c|c|}
\hline \multirow[t]{3}{*}{ Outcome } & \multirow[t]{3}{*}{ Variable } & \multicolumn{8}{|c|}{ 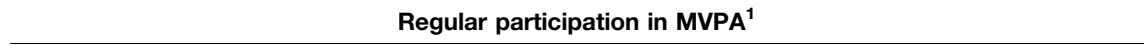 } \\
\hline & & \multicolumn{4}{|c|}{ No } & \multicolumn{4}{|c|}{ Yes } \\
\hline & & $\chi^{2}$ & $p$-value & $\mathrm{OR}^{2}$ & $d$ & $\chi^{2}$ & $p$-value & OR & $d$ \\
\hline \multirow[t]{10}{*}{ On-task behaviour } & MVPA quintile 1 & 1.84 & & 1.36 & 0.17 & 0.00 & & 0.99 & 0.00 \\
\hline & MVPA quintile 2 & 8.08 & $\star \star$ & 1.98 & 0.38 & 0.09 & & 0.93 & -0.04 \\
\hline & MVPA quintile 3 & 0.31 & & 1.57 & 0.08 & 1.36 & & 1.32 & 0.15 \\
\hline & MVPA quintile 4 & 4.44 & * & 1.71 & 0.30 & 0.27 & & 1.14 & 0.07 \\
\hline & MVPA quintile 5 & 1.23 & & 1.45 & 0.20 & 5.76 & * & 1.85 & 0.34 \\
\hline & $\mathrm{RPE}^{3} 1$ & 5.52 & * & 1.90 & 0.36 & 0.05 & & 1.06 & 0.03 \\
\hline & RPE 2 & 0.00 & & 1.00 & 0.00 & 0.11 & & 1.26 & 0.05 \\
\hline & RPE 3 & 1.69 & & 1.44 & 0.20 & 0.72 & & 1.26 & 0.13 \\
\hline & RPE 4 & 2.75 & & 1.62 & 0.27 & 2.70 & & 1.63 & 0.27 \\
\hline & RPE 5 & 0.97 & & 1.29 & 0.14 & 0.04 & & 1.06 & 0.03 \\
\hline \multirow[t]{10}{*}{ Passive off-task behaviour } & MVPA quintile 1 & 32.71 & 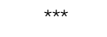 & 0.64 & -0.24 & 13.80 & $* \star \star *$ & 0.81 & -0.12 \\
\hline & MVPA quintile 2 & 21.65 & $* \star \star *$ & 0.58 & -0.30 & 20.83 & $* \star \star *$ & 0.56 & -0.32 \\
\hline & MVPA quintile 3 & 10.84 & $* * \star$ & 0.90 & -0.06 & 22.30 & 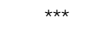 & 0.57 & -0.31 \\
\hline & MVPA quintile 4 & 6.25 & * & 1.23 & 0.11 & 11.76 & 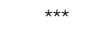 & 0.87 & -0.08 \\
\hline & MVPA quintile 5 & 3.20 & & 1.36 & 0.17 & 20.59 & $\star \star \star *$ & 0.57 & -0.31 \\
\hline & RPE 1 & 2.56 & & 0.61 & -0.27 & 0.91 & & 0.73 & -0.18 \\
\hline & RPE 2 & 0.01 & & 1.05 & 0.02 & 1.61 & & 0.65 & -0.24 \\
\hline & RPE 3 & 7.91 & ** & 0.39 & -0.52 & 1.42 & & 0.68 & -0.21 \\
\hline & RPE 4 & 1.13 & & 0.70 & -0.20 & 2.56 & & 0.57 & -0.31 \\
\hline & RPE 5 & 1.46 & & 1.43 & 0.20 & 2.09 & & 0.63 & -0.26 \\
\hline
\end{tabular}

Note: ${ }^{1}$ MVPA = Moderate-to-Vigorous Physical Activity during physical education, coded into quintiles. ${ }^{2}$ OR $=$ Odds Ratio. ${ }^{3}$ RPE $=$ Rate of Perceived Exertion, rated from 1 (not at all tiring) to 5 (extremely tiring). ${ }^{*} \mathrm{p} \leq 0.05,{ }^{* *} \mathrm{p} \leq 0.01,{ }^{* * *} \mathrm{p} \leq 0.001$. Effect sizes indicating a small ( $\left.\mathrm{d} \geq 0.2\right)$, medium ( $\mathrm{d} \geq 0.5$ ), or large (d $\geq 0.8$ ) effect are highlighted in bold font.

\subsubsection{Trait Off-Task Behaviour}

Finally, for pupils who displayed the highest levels of active and passive off-task behaviour, we found significant positive effects of all but one PE lesson intensities on on-task behaviour (Figure 5;
Table 6). For pupils high on passive disengagement, effect sizes were small $(d=0.23-0.46)$. For those high on active disengagement, effects were small to medium $(d=0.26-0.66)$. In both groups, it was noticeable that for perceived exertion the 

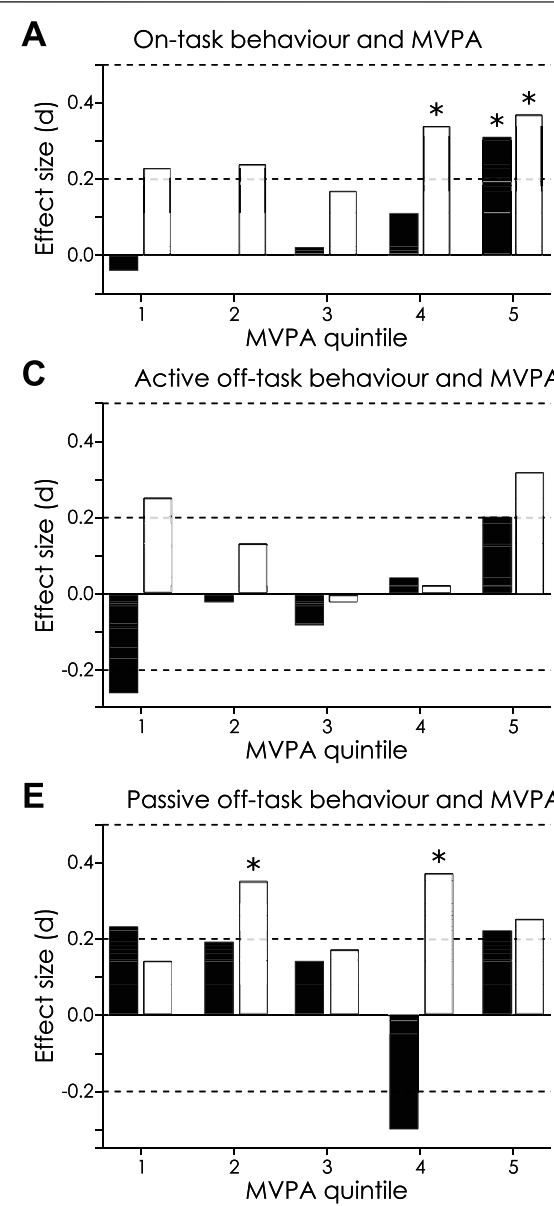
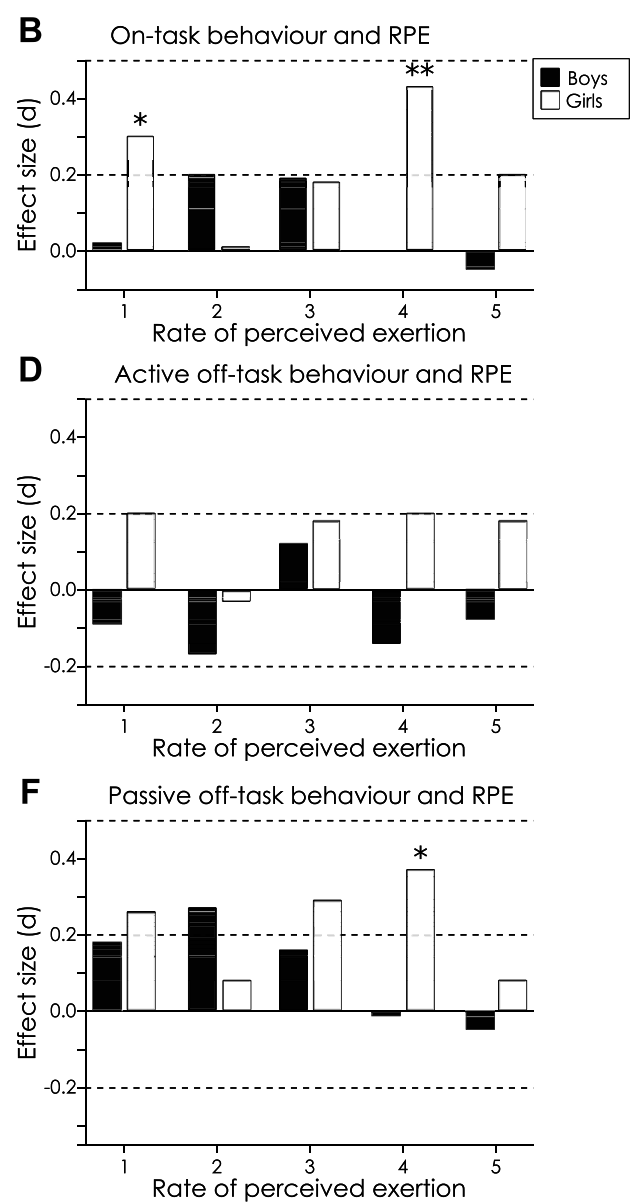

FIGURE 6 | Change in behaviour in response to objective and subjective measures of physical activity intensity, by sex. (A) On-task behaviour and MVPA (B) Ontask behaviour and RPE (C) Active off-task behaviour and MVPA (D) Active off-task behaviour and RPE (E) Passive off-task behaviour and MVPA (F) Passive off-task behaviour and RPE. Note. MVPA = Moderate-to-vigorous physical activity. Effect sizes have been transformed so that positive numbers reflect favourable effects (more on-task behaviour, less off-task behaviour). Small $(d=0.2)$, medium $(d=0.5)$, and large $(d=0.8)$ effect sizes are indicated by the dashed grid lines. ${ }^{\star} p \leq 0.05$, ${ }^{\star \star} p \leq$ 0.01 , and ${ }^{* * *} p \leq 0.001$

greatest increase in on-task behaviour happened after submaximal intensity lessons (RPE 3 and 4), with a tailing off of the effect for lessons rated at RPE 5. This tailing off was not evident in the objectively measured PA results.

\subsubsection{Sex}

We found interaction effects of sex (see Figure 6 and Table 7). In terms of on-task behaviour, boys responded only to the highest percentile of MVPA $\left(>33.5 \%, \chi^{2}=3.93, p<0.05\right.$, and $\left.d=0.31\right)$, whereas girls also experienced positive effects of lower MVPA contents (MVPA percentile 4 and $5 ; \chi^{2}=5.33, p<0.05, d=0.34$ and $\chi^{2}=5.61, p<0.05, d=0.37$, respectively, see Figure 6A). For active off-task behaviour, we found no interactions of sex (Figures 6C,D). Passive off-task behaviours were not systematically reduced in relation to subjective or objective intensity ratings in either sex (Figures 6E,F), but whereas girls' passive behaviour was reduced after a range of medium-intensity lessons $\left(\chi^{2}=4.31-4.95, p<0.05\right.$, $d=-0.35$ to -0.37 ), boys' passive off-task behaviour was not significantly affected by PA.

\subsubsection{Regular MVPA}

Those who regularly met United Kingdom government guidelines for MVPA in children (Chief Medical Officers, 2019) benefited from fewer PE lessons than those who were less regularly active (see Table 8). For active children, only after lessons with the highest MVPA content (quintile 5, $\chi^{2}=5.76, p<$ 0.05 , and $d=0.34$ ) was their on-task behaviour significantly greater. On the other hand, lesser-active children also benefited from lower-intensity lessons (MVPA quintile 2 and 4, and RPE 1, $\chi^{2}=4.44-8.08, p<0.05$, and $d=0.30-0.38$, see Figures 7A,B). Similar to sex, no interaction effect of regular MVPA was found for active off-task behaviour (Figures 7C,D). Passive off-task behaviour was lower only after low-MVPA PE lessons in lesseractive children (quintiles 1 and 2, $\chi^{2}=32.71, p<0.001, d=-0.24$ and $\chi^{2}=21.65, p<0.001, d=-0.30$, respectively), whilst in regularly active children it was reduced after every intensity lesson, with three medium to high intensity lessons reaching small effect sizes $\left(\chi^{2}=20.59-22.30, p<0.001, d=-0.31\right.$ to -0.32 , Figure 7E). Subjective intensity predicted less passive off-task 

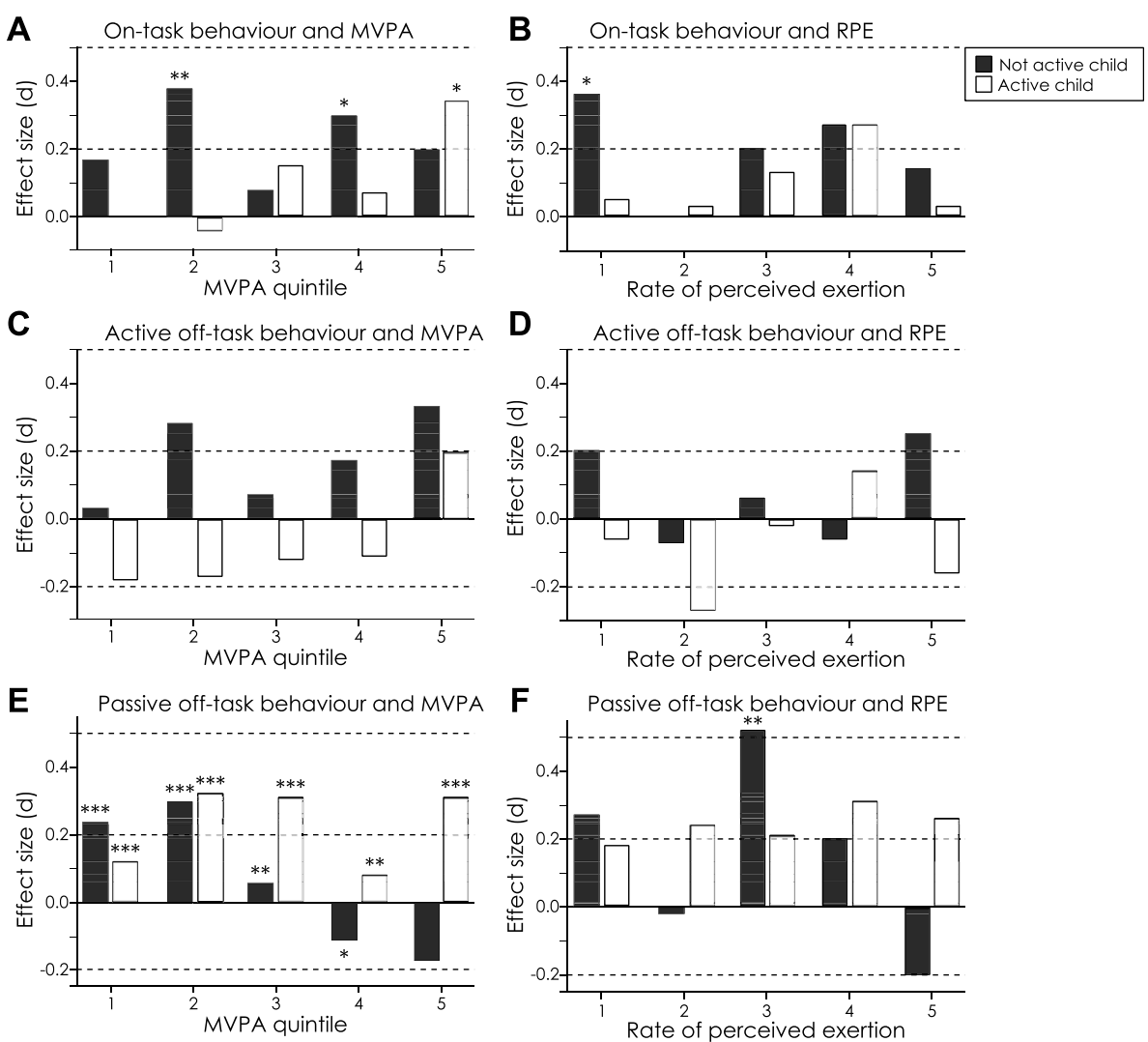

FIGURE 7 | Change in behaviour in response to objective and subjective measures of physical activity intensity, by regular moderate-to-vigorous physical activity. (A) On-task behaviour and MVPA (B) On-task behaviour and RPE (C) Active off-task behaviour and MVPA (D) Active off-task behaviour and RPE (E) Passive off-task behaviour and MVPA (F) Passive off-task behaviour and RPE. Note. MVPA = Moderate-to-vigorous physical activity. Effect sizes have been transformed so that positive numbers reflect favourable effects (more on-task behaviour, less off-task behaviour). Small $(d=0.2)$, medium $(d=0.5)$, and large $(d=0.8)$ effect sizes are indicated by the dashed grid lines. ${ }^{\star} p \leq 0.05,{ }^{\star *} p \leq 0.01$, and ${ }^{\star * \star} p \leq 0.001$.

behaviour only for lesser-active children, and only for lessons perceived to be moderately active, with a medium effect size (RPE $3, \chi^{2}=7.91, p<0.01, d=-0.52$, see Figure $\left.7 \mathbf{F}\right)$.

\subsubsection{BMI}

We found no systematic interaction effects of PA intensity and BMI category on any type of behaviour, regardless of whether objective or subjective PA measure were entered into the model.

\section{DISCUSSION}

\subsection{The On-Task Response to Physical Activity Differs Between Objectively and Subjectively Measured Activity}

In answering the first research question the results indicated that high intensity lessons had the greatest positive effect on behaviour, in line with the first part of our hypothesis. However, a difference between objective and subjective measures of PA intensity was evident. More specifically, PE lessons where the intensity was in the $4^{\text {th }}$ and $5^{\text {th }}$ quintiles of MVPA, as measured objectively with the accelerometer watches, and were found to significantly predict more ontask behaviour subsequently. However, children's subjective measures of perceived exertion were not as clear; "neutral tiring" and "little tiring" rated lessons were found to significantly affect on-task behaviour, however, with very small effect sizes $(d<0.2)$. Coupled with our finding that the high intensity designed lessons also significantly improved subsequent on-task behaviour, the results confirm previous findings that high intensity PA interventions are optimal for improving task-related behaviour (Ma et al., 2014; Szabo-Reed et al., 2017). Subjective measures of PA intensity did not align with the objective measures. The incongruence in these findings could be ascribed to children's inaccuracy in estimating the intensity of their PE lesson; children who are more active may not perceive high intensity lessons as tiring, or lower-fit children may rate lessons with less PA as highly intense. Schneider and Schmalbach (2015) found that adolescents $(M$ age $=11.09$ years $)$ who were fitter recorded lower RPE at higher heart rates during exercise than those who were less fit. 


\subsection{Passive Off-Task Behaviours Are More Responsive to Low-Intensity Physical Activity Than Active Off-Task Behaviours}

The second research question aimed to explore the effects of PA on active and passive off-task behaviour. The findings suggest that PE lessons with MVPA content in the $5^{\text {th }}$ quintile reduced subsequent active off-task behaviour in the classroom confirming the hypothesised direction of effects. However, no significant effects where found for children's perceived exertion and active off-task behaviour. The pattern for passive off-task behaviour differed from the response in active off-task behaviour; a significantly reduction was found after PE lessons where the content intensity was in the $2^{\text {nd }}$ quintile (low-to-moderate intensity). In relation to subjectively measured PA intensity, lower-intensity lessons were also most effective in reducing passive off-task behaviours, with "not at all tiring" and "neutral tiring" leading to significant reductions. Although we had expected the greatest effect on passive behaviour for moderate intensity (Unsworth and Robinson, 2020), our hypothesis that active behaviours are reduced by higher intensity activity than passive behaviours was confirmed. However, a previous study (MullenderWijnsma et al., 2015) did not find an association between MVPA and time-on-task in post-intervention lessons. These opposing results may be due to their intervention being classroom-based, whereas our took place during PE lessons. In addition, Mullender-Wijnsma et al. (2015) did not differentiate between passive and active off-task behaviour in their observation tool, therefore, the present analysis goes beyond previous literature. An important contribution of the findings is the importance of distinguishing between passive and active off-task behaviour, and that a different response in children's behaviour can be expected depending on the intensity of the PE lesson.

\subsection{Children Respond Differently to Physical Activity Intervention}

Our data confirm that children respond differently to PA depending on their individual characteristics. Below we discuss the differences in their response based on their level of on-task or specific off-task behaviour before the PE lesson, their sex, and habitual engagement in MVPA.

\subsubsection{The Least On-Task Pupils Become Less Disruptive}

As hypothesised, and in line with Mahar et al. (2006) among others, we found that those who have the lowest level of trait on-task behaviour responded most favourably to the intervention, improving their on-task behaviour regardless of the PE lesson content. Those who were mostly actively off-task responded with a small to moderate increase in on-task behaviour. On the other hand, the on-task behaviour of those who were mostly passively off-task increased only with small effect size. Thus, although for the whole sample, passive off-task behaviours appear to be most responsive to PA intervention (Figure 3), pupils who are most actively disengaged - and potentially disruptive to their peers - most strongly increased their on-task behaviour in response to PA (Figure 5). What appears to be most beneficial for these pupils is that they have the opportunity to be active, and preferably moderateto-vigorously so, before they engage in their classroom tasks.

\subsubsection{Girls Respond to a Greater Range of Activity Intensities Than Boys}

Our findings with regard to sex did not confirm our hypothesis, and are opposite also to those by Ma et al. (2014), who's results indicated greater effects of PA breaks for boys than girls. In particular, Ma et al. (2014) found that active off-task behaviours were greatly reduced in boys. In our study, no significant effects of PA of any intensity on active off-task behaviour were found for either sex. With regard to on-task behaviour, we found that girls responded to a wider range of higher-intensity (MVPA quintile 4 and 5) than boys (MVPA quintile 5 only). the same was evident for passive off-task behaviour, where girls responded positively to low-to-moderate PE lessons as well as moderate-to-high intensity lessons, whereas boys' behavioural response did not reach significance at all. It may be that due to the generally lower levels of PA in girls (van Sluijs et al., 2021), their response to lower-intensity PA is greater than in boys. Moreover, the results from Ma et al. (2014) were obtained after a 10-min activity in the classroom, rather than a full PE lesson. Thus, it may be that the sustained nature of the exercise for an entire PE lesson induces greater effects in girls, even at lower intensities, if they are less accustomed to high levels of PA than boys. The metaanalysis off school-based PA interventions for girls by Owen et al. (2017) did include a range of PA interventions, but they investigated only results for girls, rather than compare these to boys' results. Their conclusion that PA interventions for girls yield very small to small effects (Owen et al., 2017) does correspond with the findings of this study, as all effect sizes for girls found here were smaller than $d=0.5$.

\subsubsection{Inactive Children Become More On-Task and Less Passively Off-Task After Low-To-Medium, but Not High-Intensity Physical Activity}

We hypothesised that more regularly active children would benefit more greatly from the intervention. Our results did not confirm this hypothesis, as we found that the results differed, based on which behaviour was specified as the outcome variable. When on-task behaviour was examined, lesser-active children responded favourably to a range of lower-intensity lessons, and whereas more regularly fit children only benefited from lessons with the highest MVPA content. It may be that children who do not regularly engage in MVPA have a stronger physiological response to the MVPA content of PE lessons, thus benefiting from lower-intensity lessons than those who do regularly engage in MVPA. When we examined passive off-task behaviours, the 
same pattern was found, with regularly active children responding more favourably to higher MVPA content than lesser-active children. Our findings are in line with Snyder et al. (2017), who found significant reductions in passive offtask behaviour. Our results further demonstrate the importance of incorporating intensity levels in intervention designs of this nature, to establish their differential effects. However, for this outcome variable, regularly active children had a significant $(p<$ $0.05)$ and meaningful $(d \geq 0.2)$ response to three of the PE lesson MVPA quintiles, versus only two for the lesser-active sub-sample. We therefore can conclude that children respond differently to PA intensity based on their habitual engagement in MVPA, but we cannot say that lesser-active or higher-active children respond more favourably to PA overall.

\subsubsection{BMI Does Not Interact With PE Lesson Intensity}

Our hypothesis regarding the effect of BMI was confirmed; we did not find systematic interaction effects of BMI and PA intensity. This is in line with the review by Chang et al. (2017), reporting inconclusive moderation effects of BMI on the relationship between $\mathrm{PA}$ and cognitive performance. Similarly, our results correspond with the findings of the meta-analysis by Sun et al. (2021), which details effects for PA in overweight and obese samples that are of a similar magnitude to what has previously been reported in samples of healthy-weight children or mixed samples (e.g., Owen et al., 2016; De Greeff et al., 2018). Specific to the acute effects of PA on task-behaviour in a classroom context, our results also correspond with those from Grieco et al. (2016), who found that BMI only interacted with PA when activity was compared to inactive classroom lessons, and not with PA intensity levels. As this study compared PE lessons of various intensities, without an inactive control group, and the absence of an interaction effect for BMI is in line with the current literature.

\subsection{Strengths and Limitations}

The conclusion drawn from the results are validated by the ecological validity of the study. The intervention was designed so as to be implemented with minimal disruption to the "business as usual" lessons. The nature of observations was also such that the trained observers did not interfere with the class teacher and the lesson, so that children's learning, and behaviour was minimally affected. Furthermore, the PE lessons themselves were carefully designed to reflect the national curriculum for $\mathrm{PE}$ in the United Kingdom, and delivered in the same manner to every class. The rigorous methodological design ensured a detailed and intensive observational measure which enabled the researchers to collect a large number of observations or data points suitable for the analysis conducted. An additional strength of the study was its within-person design, where all participants took part in all the different PE intervention lessons, allowing the analysis of both within- and between-person variance. The study also had certain limitations. For example, a smaller sub-sample was used to answer the third research question, as the sample was split into smaller sub-samples. This may have contributed to the absence of effects for BMI, as the sample was split across four categories, rather than the two categories in analyses of interactions of regular MVPA and sex. While the findings from our study are promising, a larger scale replication would be beneficial, with a similar study design and distinction between levels of intensity and different types of offtask behaviour, which is sparse in the wider literature. Finally, it must be noted that in observation studies, a certain level of inference cannot be avoided. Moreover, it was not possible to blind the observers to the conditions of the PE lesson. To address these limitations and ensure the observations were of high quality, one of the two observers was blind to the hypotheses of the study, and inter-rater reliability checks were regularly carried out throughout the data collection period, with Cohen's Kappa $>0.8$ at all times.

\subsection{Implications}

The present findings further add to the wider literature in emphasising the importance of PA in school and its potential subsequent positive effect on children's learning behaviour. Completing the required class-work demands a level of focus and concentration, which can be facilitated if on-task behaviour can be increased. These results also have direct implications for primary school teachers, as they can use the content of PE lessons to support their pupils' learning in the classroom, and in particular the behaviour of the most disruptive pupils. Moreover, our finding that children's improvement in on-task behaviour is dependant on whether they are actively or passively off-task, is an important one. Teachers' awareness of the type of off-task behaviour their class is exhibiting can enable them to better tailor the intensity of $\mathrm{PE}$ lessons for optimal effectiveness. Furthermore, individual differences are important for teachers to take into account, notably children's PA levels outside of school. The lesser-active children in our sample did not benefit from the highestintensity lessons, which they rated as highly tiring. Therefore, a substantial implication of the findings is that $\mathrm{PE}$ lessons and PA breaks during learning are not a "onesize-fits-all", and positive effects can be maximised if the intensity level is adjusted to the individual child.

\subsubsection{Conclusion}

The present study aimed to investigate the effects of different intensity PE lessons on on- and off-task behaviour in the classroom in primary school-aged children. The results have demonstrated that individual differences in children play a role in their response to different types of PE lessons. By distinguishing between different intensities of PA, the results demonstrated the nuance necessary in furthering our understanding of the effects of PA on on-task behaviour. Overall, the findings emphasise the importance of the positive 
effects of PE on children's classroom behaviour. Our findings, in line with the wider literature, reinforce the importance for schools to expand, extend, and enhance opportunities for PA in the curriculum.

\section{DATA AVAILABILITY STATEMENT}

The raw data supporting the conclusion of this article will be made available by the authors, without undue reservation.

\section{ETHICS STATEMENT}

The studies involving human participants were reviewed and approved by Oxford University Department of Education

\section{REFERENCE}

Álvarez-Bueno, C., Pesce, C., Cavero-Redondo, I., Sánchez-López, M., MartínezHortelano, J. A., and Martínez-Vizcaíno, V. (2017). The Effect of Physical Activity Interventions on Children's Cognition and Metacognition: A Systematic Review and Meta-Analysis. J. Am. Acad. Child Adolesc. Psychiatry 56 (9), 729-738. doi:10.1016/j.jaac.2017.06.012

Ayan, C., Cancela Carral, J., and Montero, C. (2014). Academic Performance of Young Competitive Swimmers Is Associated with Physical Activity Intensity and its Predominant Metabolic Pathway: A Pilot Study. J. Phys. Act Health 11 (7), 1415-1419. doi:10.1123/jpah.2012-0453

Beets, M. W., Okely, A., Weaver, R. G., Webster, C., Lubans, D., Brusseau, T., et al. (2016). The Theory of Expanded, Extended, and Enhanced Opportunities for Youth Physical Activity Promotion. Int. J. Behav. Nutr. Phys. Act 13, 120. doi:10.1186/s12966-016-0442-2

Benzing, V., Heinks, T., Eggenberger, N., and Schmidt, M. (2016). Acute Cognitively Engaging Exergame-Based Physical Activity Enhances Executive Functions in Adolescents. PLoS One 11, e0167501. doi:10.1371/journal.pone.0167501

Buchan, D. S., Ollis, S., Thomas, N. E., Buchanan, N., Cooper, S. M., Malina, R. M., et al. (2011). Physical Activity Interventions: Effects of Duration and Intensity. Scand. J. Med. Sci. Sports 21 (6), e341-50. doi:10.1111/j.1600-0838.2011.01303.x

Burns, R. D., Fu, Y., and Podlog, L. W. (2017). School-based Physical Activity Interventions and Physical Activity Enjoyment: A Meta-Analysis. Prev. Med. 103 (May), 84-90. doi:10.1016/j.ypmed.2017.08.011

Chang, Y. K., Chu, C. H., Chen, F. T., Hung, T. M., and Etnier, J. L. (2017). Combined Effects of Physical Activity and Obesity on Cognitive Function: Independent, Overlapping, Moderator, and Mediator Models. Sports Med. 47 (3), 449-468. doi:10.1007/s40279-016-0589-7

Charlton, C., Rasbash, J., Browne, W. J., Healy, M., and Cameron, B. (2019). Mlwin Version 3.04 (Computer Program). Bristol: Centre for Multilevel Modelling, University of Bristol, Bristol. Available at: http://www.bristol.ac.uk/cmm/ software/mlwin/download/.

Chief Medical Officers (2019). Uk Chief Medical Officers' Physical Activity Guidelines [Guidance from the Chief Medical Office. Available at: https:// assets.publishing.service.gov.uk/government/uploads/system/uploads/ attachment_data/file/832868/ukchief- medical-officers-physical-activityguidelines.pdf

Cole, T. J., Flegal, K. M., Nicholls, D., and Jackson, A. A. (2007). Body Mass index Cut Offs to Define Thinness in Children and Adolescents: International Survey. BMJ 335, 194. doi:10.1136/bmj.39238.399444.55

de Bruijn, A. G. M., Kostons, D. D. N. M., Van Der Fels, I. M. J., Visscher, C., Oosterlaan, J., Hartman, E., et al. (2020). Effects of Aerobic and CognitivelyEngaging Physical Activity on Academic Skills: A Cluster Randomized
Departmental Research Ethics Committee. Written informed consent to participate in this study was provided by the participants' legal guardian/next of kin.

\section{AUTHOR CONTRIBUTIONS}

$\mathrm{CH}$ : Conceptualisation; Data curation; Formal analysis; Investigation; Methodology; Project administration; Writing-original draft; Writing-review and editing. MS: Data curation; Investigation; Writing-original draft; Writing-review and editing. SS: Supervision; Conceptualisation; Methodology. LM: Supervision; Conceptualisation; Data curation; Formal analysis Methodology; Writing-review and editing.

Controlled Trial. J. Sports Sci. 38 (15), 1806-1817. doi:10.1080/ 02640414.2020.1756680

De Greeff, J. W., Bosker, R. J., Oosterlaan, J., Visscher, C., and Hartman, E. (2018). Effects of Physical Activity on Executive Functions, Attention and Academic Performance in Preadolescent Children: A Meta-Analysis. J. Sci. Med. Sport 21 (5), 501-507. doi:10.1016/j.jsams.2017.09.595

De Greeff, J. W., Hartman, E., Mullender-Wijnsma, M. J., Bosker, R. J., Doolaard, S., and Visscher, C. (2016). Effect of Physically Active Academic Lessons on Body Mass index and Physical Fitness in Primary School Children. J. Sch. Health 86 (5), 346-352. doi:10.1111/josh.12384

de Onis, M., Onyango, A. W., Borghi, E., Siyam, A., Nishida, C., and Siekmann, J. (2007). Development of a Who Growth Reference for School-Aged Children and Adolescents. Bull. World Health Organ. 85, 660-667. doi:10.2471/ blt.07.043497

Egger, F., Benzing, V., Conzelmann, A., and Schmidt, M. (2019). Boost Your Brain, while Having a Break! the Effects of Long-Term Cognitively Engaging Physical Activity Breaks on Children's Executive Functions and Academic Achievement. PloS one 14 (3), e0212482. doi:10.1371/ journal.pone.0212482

Egger, F., Conzelmann, A., and Schmidt, M. (2018). The Effect of Acute Cognitively Engaging Physical Activity Breaks on Children's Executive Functions: Too Much of a Good Thing? Psychol. Sport Exerc. 36 (100), 178-186. doi:10.1016/j.psychsport.2018.02.014

Erickson, K. I., Hillman, C. H., and Kramer, A. F. (2015). Physical Activity, Brain, and Cognition. Curr. Opin. Behav. Sci. 4, 27-32. doi:10.1016/ j.cobeha.2015.01.005

Fredricks, J. A., Blumenfeld, P. C., and Paris, A. H. (2004). School Engagement: Potential of the Concept, State of the Evidence. Rev. Educ. Res. 74 (1), 59-109. doi:10.3102/00346543074001059

Godwin, K., Almeda, M., Petroccia, M., Baker, R., and Fisher, A. (2013). Classroom Activities and Off-Task Behavior in Elementary School Children.

Godwin, K., and Fisher, A. (2011). Allocation of Attention in Classroom Environments: Consequences for Learning.

Goh, T. L., Hannon, J., Webster, C., and Podlog, L. (2016). Effects of a Take 10! Classroom-Based Physical Activity Intervention on Third- to Fifth-Grade Children's On-Task Behavior. J. Phys. Activity Health 13, 712-718. doi:10.1123/jpah.2015-0238

Goh, T. L., Fu, Y., Brusseau, T., and Hannon, J. (2018). On-task Behavior of Elementary Students during Movement Integration. J. Phys. Edu. Sport 18 (1), 103-106. doi:10.7752/jpes.2018.01013

Grieco, L. A., Jowers, E. M., Errisuiz, V. L., and Bartholomew, J. B. (2016). Physically Active vs. Sedentary Academic Lessons: A Dose Response Study for Elementary Student Time on Task. Prev. Med. 89, 98-103. doi:10.1016/ j.ypmed.2016.05.021 
Groslambert, A., Hintzy, F., Hoffman, M. D., Dugué, B., and Rouillon, J. D. (2001). Validation of a Rating Scale of Perceived Exertion in Young Children. Int. J. Sports Med. 22 (2), 116-119. doi:10.1055/s-2001-11340

Heemskerk, C. H., Lubans, D., Strand, S., and Malmberg, L.-E. (2019). The Effect of Physical Education Lesson Intensity and Cognitive Demand on Subsequent Learning Behaviour. J. Sci. Med. Sport 23 (6), 586-590. doi:10.1016/ j.jsams.2019.12.012

Heemskerk, C. H., and Malmberg, L.-E. (2020). Students' Observed Engagement in Lessons, Instructional Activities, and Learning Experiences. Frontline Learn. Res. 8 (6), 38-58. doi:10.14786/flr.v8i6.613

Hildebrand, M., Van Hees, V., Hansen, B. H., and Ekelund, U. (2014). Age Group Comparability of Raw Accelerometer Output from Wrist- and Hip-Worn Monitors. Med. Sci. Sports Exerc. 46 (9), 1816-1824. doi:10.1249/ mss.0000000000000289

IBM Corporation (2020). Ibm Spss Version 27.0 (Computer Program). Armonk, NY: IBM Corporation.

Inchley, J., Currie, D., Budisavljevic, S., Torsheim, T., Jåstad, A., Cosma, A., et al. (2020). Spotlight On Adolescent Health and Well-Being: Findings From the 2017/ 2018 Health Behaviour in School-Aged Children (Hbsc) Survey in Europe and canada. tech. rep. No. 9789289055017. Copenhagen, Denmark: World Health Organisation, Regional Office for Europe. https://apps.who.int/iris/handle/ $10665 / 332104$.

Ishihara, T., Sugasawa, S., Matsuda, Y., and Mizuno, M. (2017). Improved Executive Functions in 6-12-Year-Old Children Following Cognitively Engaging Tennis Lessons. J. Sports Sci. 35 (20), 2014-2020. doi:10.1080/02640414.2016.1250939

Jäger, K., Schmidt, M., Conzelmann, A., and Roebers, C. M. (2015). The Effects of Qualitatively Different Acute Physical Activity Interventions in Real-World Settings on Executive Functions in Preadolescent Children. Ment. Health Phys. Activity 9. doi:10.1016/j.mhpa.2015.05.002

Jurakić, D., and Pedišić, Ž. (2013). The Prevalence of Insufficient Physical Activity in Children and Adolescents. Peadiatria Croatica 56 (4).

Kubesch, S., Walk, L., Spitzer, M., Kammer, T., Lainburg, A., Heim, R., et al. (2009). A 30-minute Physical Education Program Improves Students' Executive Attention. Mind, Brain Edu. 3 (4), 235-242. doi:10.1111/j.1751-228X.2009.01076.x

Kybartas, T. J., Oody, J. F., Fairbrother, J. T., Durham, S., and Coe, D. P. (2021). Physical Activity Intensity, Self-Regulation, and School Readiness Indicators in Young Children. Early Child. Dev. Care 191 (4), 501-510. doi:10.1080/ 03004430.2019 .1625896

Lubans, D., Richards, J., Hillman, C., Faulkner, G., Beauchamp, M., Nilsson, M., et al. (2016). Physical Activity for Cognitive and Mental Health in Youth: A Systematic Review of Mechanisms. Pediatrics 138 (3), 1642-1656. doi:10.1542/ peds.2016-1642

Ma, J. K., Mare, L. L., and Gurd, B. J. (2014). Classroom-based High-Intensity Interval Activity Improves Off-Task Behaviour in Primary School Students. Appl. Physiol. Nutr. Metab. 39 (12), 1332-1337. doi:10.1139/apnm-2014-0125

Mahar, M. (2019). Classroom-based Physical Activity and On-Task Behavior. Translational J. Am. Coll. Sports Med. 4 (17), 148-154. doi:10.1249/TJX.0000000000000099

Mahar, M. (2011). Impact of Short Bouts of Physical Activity on Attention-ToTask in Elementary School Children. Prev. Med. 52, S60-S64. doi:10.1016/ j.ypmed.2011.01.026

Mahar, M., Murphy, S. K., Rowe, D. A., Golden, J., Shields, A. T., and Raedeke, T. D. (2006). Effects of a Classroom-Based Program on Physical Activity and OnTask Behavior. Med. Sci. Sports Exerc. 12, 2086-2094. doi:10.1249/ 01.mss.0000235359.16685.a3

Martin, A., Booth, J., Laird, Y., Sproule, J., Reilly, J. J., and Saunders, D. H. (2018). Physical Activity, Diet and Other Behavioural Interventions for Improving Cognition and School Achievement in Children and Adolescents with Obesity or Overweight. Cochrane Database Syst. Rev. (3). doi:10.1002/ 14651858.CD009728.pub410.1002/14651858.CD009728.pub3

Masini, A., Marini, S., Gori, D., Leoni, E., Rochira, A., and Dallolio, L. (2020). Evaluation of School-Based Interventions of Active Breaks in Primary Schools: A Systematic Review and Meta-Analysis. J. Sci. Med. Sport 23 (4), 377-384. doi:10.1016/j.jsams.2019.10.008

Mavilidi, M. F., Drew, R., Morgan, P. J., Lubans, D. R., Schmidt, M., and Riley, N. (20191992). Effects of Different Types of Classroom Physical Activity Breaks on
Children's On-Task Behaviour and Academic Achievement, and Cognition. Acta Paediatr. (Oslo, Norway. doi:10.1111/apa.14892

Mavilidi, M. F., Mason, C., Leahy, A. A., Kennedy, S. G., Eather, N., Hillman, C. H., et al. (2021). Effect of a Time-Efficient Physical Activity Intervention on Senior School Students' On-Task Behaviour and Subjective Vitality: The 'burn 2 Learn' Cluster Randomised Controlled Trial. Educ. Psychol. Rev. 33 (1), 299-323. doi:10.1007/s10648-020-09537-x

Maykel, C., Bray, M., and Rogers, J. H. (2018). A Classroom-Based Physical Activity Intervention for Elementary Student On-Task Behavior. J. Appl. Sch. Psychol. 34 (3), 259-274. doi:10.1080/15377903.2017.1403402

Meijer, A., Königs, M., de Bruijn, A. G. M., Visscher, C., Bosker, R. J., Hartman, E., et al. (2021). Cardiovascular Fitness and Executive Functioning in Primary School-Aged Children. Dev. Sci. 24 (2). doi:10.1111/desc.13019

Migueles, J., Rowlands, A., Huber, F., Sabia, S., and Van Hees, V. (2019). Ggir: A Research Community-Driven Open Source R Package for Generating Physical Activity and Sleep Outcomes from Multi-Day Raw Accelerometer Data. J. Meas. Phys. Behav. (2), 188-196. doi:10.1123/jmpb.2018-0063

Mora-Gonzalez, J., Esteban-Cornejo, I., Cadenas-Sanchez, C., Migueles, J. H., Molina-Garcia, P., Rodriguez-Ayllon, M., et al. (2019). Physical Fitness, Physical Activity, and the Executive Function in Children with Overweight and Obesity. J. Pediatr., 50-56. doi:10.1016/j.jpeds.2018.12.028

Mullender-Wijnsma, M. J., Hartman, E., de Greeff, J. W., Bosker, R. J., Doolaard, S., and Visscher, C. (2015). Moderate-to-vigorous Physically Active Academic Lessons and Academic Engagement in Children with and without a Social Disadvantage: A within Subject Experimental Design. BMC Public Health 15. doi:10.1186/s12889-015-1745-y

Owen, K. B., Parker, P. D., Van Zanden, B., MacMillan, F., Astell-Burt, T., and Lonsdale, C. (2016). Physical Activity and School Engagement in Youth: A Systematic Review and Meta-Analysis. Educ. Psychol. 51 (2), 129-145. doi:10.1080/00461520.2016.1151793

Owen, M. B., Curry, W. B., Kerner, C., Newson, L., and Fairclough, S. J. (2017). The Effectiveness of School-Based Physical Activity Interventions for Adolescent Girls: A Systematic Review and Meta-Analysis. Prev. Med. 105, 237-249. doi:10.1016/j.ypmed.2017.09.018

Rane, S. (2017). Emojione (Computer Program). https://www.emojione.com/.

Schneider, M., and Schmalbach, P. (2015). Affective Response to Exercise and Preferred Exercise Intensity Among Adolescents. J. Phys. Activity Health 12 (4), 546-552. doi:10.1123/jpah.2013-0442

Sember, V., Jurak, G., Kovač, M., Morrison, S. A., and Starc, G. (2020). Children's Physical Activity, Academic Performance, and Cognitive Functioning: A Systematic Review and Meta-Analysis. Front. Public Health 8 (307). doi:10.3389/fpubh.2020.00307

Snyder, K., Dinkel, D., Schaffer, C., Hiveley, S., and Colpitts, A. (2017). Purposeful Movement: The Integration of Physical Activity into a Mathematics Unit. Int. J. Res. Edu. Sci. 3 (1), 75-87. doi:10.21890/IJRES.24378

Steinlin, M. (2007). The Cerebellum in Cognitive Processes: Supporting Studies in Children. Cerebellum 6 (3), 237-241. doi:10.1080/ 14734220701344507

Sun, X., Li, Y., Cai, L., and Wang, Y. (2021). Effects of Physical Activity Interventions on Cognitive Performance of Overweight or Obese Children and Adolescents: A Systematic Review and Meta-Analysis. Pediatr. Res. 89 (1), 46-53. doi:10.1038/s41390-020-0941-3

Syväoja, H. J., Kankaanpää, A., Kallio, J., Hakonen, H., Kulmala, J., Hillman, C. H., et al. (2018). The Relation of Physical Activity, Sedentary Behaviors, and Academic Achievement Is Mediated by Fitness and Bedtime. J. Phys. Activity Health 15 (2), 135-143. doi:10.1123/jpah.2017-0135

Szabo-Reed, A. N., Willis, E. A., Lee, J., Hillman, C. H., Washburn, R. A., and Donnelly, J. E. (2017). Impact of Three Years of Classroom Physical Activity Bouts on Time-On-Task Behavior. Med. Sci. Sports Exerc. 49 (11), 2343-2350. doi:10.1249/MSS.0000000000001346

Unsworth, N., and Robinson, M. K. (2020). Working Memory Capacity and Sustained Attention: A Cognitive-Energetic Perspective. J. Exp. Psychol. Learn. Mem. Cogn. 46 (1), 77-103. doi:10.1037/xlm0000712

van Sluijs, E. M. F., Ekelund, U., Crochemore-Silva, I., Guthold, R., Ha, A., Lubans, D. L., et al. (2021). Physical Activity Behaviours in Adolescence: Current 
Evidence and Opportunities for Intervention. The Lancet 398 (10298), 429-442. doi:10.1016/S0140-6736(21)01259-9

Vanderwall, C., Eickhoff, J., Randall Clark, R., and Carrel, A. L. (2018). Bmi Z-Score in Obese Children Is a Poor Predictor of Adiposity Changes over Time. BMC Pediatr. 18 (1). doi:10.1186/s12887-018-1160-5

Vazou, S., and Smiley-Oyen, A. (2014). Moving and Academic Learning Are Not Antagonists: Acute Effects on Executive Function and Enjoyment. J. Sport Exerc. Psychol. 36 (5), 474-485. doi:10.1123/jsep.2014-0035

Vogan, V. M., Leung, R. C., Safar, K., Martinussen, R., Smith, M. L., and Taylor, M. J. (2018). Children with Asd: Relations with Social, Emotional, and Behavioral Functioning over Time. Front. Psychol. 9. doi:10.3389/ fpsyg.2018.01774

Wilson, A., Olds, T., Lushington, K., Petkov, J., and Dollman, J. (2016). The Impact of 10-minute Activity Breaks outside the Classroom on Male Students' On-Task Behaviour and Sustained Attention: A Randomised Crossover Design. Acta Paediatr. 105 (4), e181-e188. doi:10.1111/ apa.13323
Conflict of Interest: The authors declare that the research was conducted in the absence of any commercial or financial relationships that could be construed as a potential conflict of interest.

Publisher's Note: All claims expressed in this article are solely those of the authors and do not necessarily represent those of their affiliated organizations, or those of the publisher, the editors and the reviewers. Any product that may be evaluated in this article, or claim that may be made by its manufacturer, is not guaranteed or endorsed by the publisher.

Copyright (๑ 2022 Heemskerk, Sumatic, Strand and Malmberg. This is an openaccess article distributed under the terms of the Creative Commons Attribution License (CC BY). The use, distribution or reproduction in other forums is permitted, provided the original author(s) and the copyright owner(s) are credited and that the original publication in this journal is cited, in accordance with accepted academic practice. No use, distribution or reproduction is permitted which does not comply with these terms. 